\title{
Honeybee gut microbiota modulates host behaviors and
}

\section{neurological processes}

Zijing Zhang ${ }^{1}$, Xiaohuan $\mathrm{Mu}^{1}$, Qina $\mathrm{Cao}^{1}$, Yao Shi ${ }^{1}$, Xiaosong $\mathrm{Hu}^{1}$, Hao Zheng ${ }^{1,2, *}$

${ }^{1}$ College of Food Science and Nutritional Engineering, China Agricultural University, 100083

Beijing, China

*e-mail: hao.zheng@cau.edu.cn (hao.zheng@cau.edu.cn). 


\section{Abstract}

Honeybee is a highly social insect with a reach behavioral repertoire and is a versatile model for neurobiological research. The honeybee gut microbiota is composed of a limited number of bacterial phylotypes that play an important role in host health. However, it remains unclear whether the microbiota can shape brain profiles and behaviors. Here, we revealed that the gut microbiota is requisite for the olfactory learning and memory ability of honeybees and alters the level of neurotransmitters in the brain. Transcriptomic and proteomic analysis showed distinctive gene expression and protein signatures for gnotobiotic bees associated with different gut bacteria. Specifically, genes related to olfactory functions and labor division are most upregulated. Moreover, differentially spliced genes in the brains of colonized bees largely overlapped with the datasets for human autism. The circulating metabolome profiles identified that different gut species regulated specific module of metabolites in the host hemolymph. Most altered metabolites are involved in the amino acid and glycerophospholipid metabolism pathways for the production of neuroactive compounds. Finally, antibiotic treatment disturbed the gut community and the nursing behavior of worker bees under field conditions. The brain transcripts and gut metabolism was also greatly interfered in treated bees. Collectively, we demonstrate that the gut microbiota regulates honeybee behaviors, brain gene transcription, and the circulating metabolism. Our findings highlight the contributions of honeybee gut microbes in the neurological processes with striking parallels to those found in other animals, thus providing a promising model to understand the host-microbe interactions via the gut-brain axis.

Keywords: Apis mellifera, gut microbiota, social behavior, metabolism, gut-brain axis 


\section{Introduction}

There is growing recognition that the gut microbiota plays a significant role in

modulating host development and physiology, including metabolism and immune functions.

Recent researches have focused on the effects of symbiotic microbes on the host's central

nervous system (CNS) and their involvement in the host behavioral processes. It reveals that

gut microbiota can impact the host brain through a diverse set of pathways such as immune

modulation and production of microbial metabolites implicated in the regulation of the gut-

brain axis ${ }^{1,2}$. The symbiotic microorganisms inhabiting the gastrointestinal intestine are

capable of producing various metabolites including neurotransmitters, amino acids, and short-chain fatty acids (SCFAs) that influence brain physiology $y^{3-5}$. While it is unclear if the neurotransmitters produced by certain gut bacteria (e.g., GABA, serotonin, and dopamine) can reach the brain considering their short half-lives and the block by the blood-brain barrier, the gut microbiota is capable of influencing brain physiology indirectly. Various SCFAs derived from microbial fermentation in the gut such as propionate and butyrate were suggested to regulate the rate-limiting enzymes involved in the biosynthesis of neurotransmitters in the brain ${ }^{6}$. Gut microbiota interferes CNS serotonergic neurotransmission by downregulating the level of tryptophan, the precursor of 5hydroxytryptamine (5-HT), in the circulatory system, which further reduced the anxiety behavior in germ-free mice ${ }^{5}$. Furthermore, the expression level and alternative splicing of autism spectrum disorder (ASD)-related genes in the brain are proudly disturbed in mice harboring human ASD microbiome, which produces differential metabolome profiles ${ }^{7}$.

44 Although the significance of the functional connection between microbiota and neurophysiology has been widely appreciated, most current studies focused on the 
compositions of gut community and the difficulty to maintain and manipulate gnotobiotic animals ${ }^{8}$. Thus, models exhibiting high sociality and less complex gut community would be ideal to fully understand the relationship between the gut microbiota and host social behaviors.

Honeybee is a eusocial insect with distinct behavioral structures characterized by a complex range of interactive behaviors within the hive, and it has been extensively used as a model of perception, cognition, and social behaviors. A set of established methods are available to quantify the sophisticated behaviors of honeybee, such as associative appetitive learning and memory, sensory responsiveness, and hive behavioral observation ${ }^{9}$. It has been well documented that honeybees have a simple and host-specialized gut microbiota, with $8 \sim$ 10 bacterial phylotypes comprising over $97 \%$ of the community ${ }^{10-12}$. Most bacterial phylotypes contain several divergent 'sequence-discrete populations' (SDPs) and a high extent of strain-level diversity ${ }^{10,12}$. All major bacterial phylotypes, including Snodgrassella, Gilliamella, Bifidobacterium, Lactobacillus Firm-4 and Firm-5, and Bartonella can be cultivated in the laboratory. Additionally, microbiota-free (MF) bees are experimentally tractable and can be colonized with defined communities of cultured strains ${ }^{13,14}$. Bee gut bacteria inhabit diverse niches and play specific roles in the bee gut, and they are beneficial to the host nutrition, immune homeostasis, and pathogen resistance ${ }^{15}$. These are probably accomplished via the microbial fermentation in the gut. The bee gut microbiota contributes to the degradation of diet polysaccharides, and untargeted metabolomics revealed that a plethora of organic acids accumulate in the presence of gut bacteria, which may have pivotal functional consequences in host physiology ${ }^{13,16}$.

Although the impact of gut community on the host's health is relatively clear, few experiments have searched for the potential links between the honeybee gut microbiota and behavior. Pioneering explorations find that the level of biogenic amines (serotonin, 
dopamine, octopamine) implicated in bee behaviors is lower in newly emerged bees, which have an immature gut community ${ }^{17}$. In-lab-generated bees with a conventional (CV) gut microbiota behave differently in the gustatory responsiveness, and they possess altered endocrine signaling compared to the MF bees. Indeed, the gut microbiota affect the host metabolism that the hemolymph metabolomic profiles of CV and MF bees are separated ${ }^{14}$. The mono-association with the Bifidobacterium asteroides elevates the concentration of juvenile hormone III derivatives in the gut, which may regulate the host development ${ }^{13}$. All these findings strongly suggest that the honeybee gut microbiota may contribute to the host brain physiology and behavior phenotypes. Thus, it provides a particularly well-suited model to gain a detailed understanding of the gut microbiota-brain interactions. with a conventional microbiota and identified that the presence of microbiota was sufficient to promote the host's perception and cognition. Multi-omics analysis revealed that gut

86 bacteria impact the neurotransmitter concentration, transcriptional program, protein level in 87 the brain, as well as the circulating metabolic profiles. Finally, we confirmed that antibiotic exposure under field condition disturbs the hive behaviors of nurse bees, which is associated with altered brain transcripts and metabolite pools in the gut. 


\section{Gut microbiota alters honeybee behaviors and brain neurotransmitter level}

The ability to discriminate and memorize odors is critical for the social behaviors of honeybees, such as division of labor, organization of feeding, kin recognition, and mating ${ }^{18,19}$. We first examined whether the colonization of gut microbiota affects the olfactory learning and memory ability of bees under laboratory conditions. Each individual of $\mathrm{CV}$, tetracycline treated $(\mathrm{CV}+$ tet $)$, or MF bees generated in the lab (Methods, Supplementary Fig. 1a) was trained for 10 trials to associate the stimulus odor (nonanol) to a sucrose reward, and the memory test was performed $3 \mathrm{~h}$ after the associative learning. Bees only responded to nonanol odor were considered to be a successful one (Fig. 1a, Supplementary Movie 1). Almost $50 \%$ of CV bees were able to memorize the nonanol odor and can distinguish the conditioned stimulus from the negative control odor (hexanol), and this percentage is similar to the previous test of hive bees performing the olfactory learning $\operatorname{task}^{20}$. In contrast, the proportion of successfully memorized individuals was significantly decreased in the antibiotic treatment group, and surprisingly, not an MF bee exhibited successful memory behavior (Fig. 1b). This suggests that the gut microbiota can apparently affect the learning and memory ability of bees. Proboscis extension response (PER) is a taste-related behavior that is fundamental for olfactory discrimination ${ }^{21}$. We then measured the PER of MF and CV bees, and bees mono-colonized with six different bacterial phylotypes (Snodgrassella, Sn; Gilliamella, Gi; Bifidobacterium, Bi; Lactobacillus Firm-4, F4; Lactobacillus Firm-5, F5; Bartonella, Ba) to estimate the olfactory sensation affected by

111 the specific gut member. Compared to the MF group, CV bees are more sensitive to the low 112 concentration of sucrose, which is consistent with the previous finding ${ }^{14}$. However, for the 
113 mono-colonized groups, only the colonization of F5 significantly elevated the sucrose

114 sensitivity of bees (Fig. 1c), implying an integrative effect of the gut bacteria.

115 Disordered olfactory behaviors are associated with alteration of neurotransmission in

116 the bee brain ${ }^{22,23}$. Therefore, we investigated the changes in the brain's neurochemistry of

$117 \mathrm{MF}, \mathrm{CV}$, and mono-colonized bees. The concentration of five major neurotransmitters 5-HT,

118 dopamine, GABA, tyramine, and octopamine that are important modulators of honeybee

119 behaviors were determined in the brains. The concentration of 5-HT was significantly lower

120 in CV bees and bees mono-colonized with Gi, F4, F5, and Ba than that in the MF bees (Fig.

121 1d). Likewise, dopamine that inhibits appetitive learning and decreases sucrose sensitivity in

122 foragers ${ }^{22,23}$ was also decreased in bacteria-colonized bees (Fig. 1e). In contrast, the

123 inhibitory transmitter GABA, which is required for fine odor discrimination ${ }^{24}$ and odor

124 learning 25,26 , was significantly higher in the brains of Bi, Sn, and F4 bees (Fig. 1F). The

125 biogenic amine, octopamine, and its precursor tyramine were not obviously altered by the

126 conventional gut microbiota, while they are lowered in mono-colonized bee groups

127 (Supplementary Fig. 1b, c). All these findings indicate that the colonization of either the

128 normal gut microbiota or each single core gut member can affect the neurotransmitter levels

129 in the brain, which might be associated with the altered olfactory sensitivity and learning-

130 memory performance. 
A

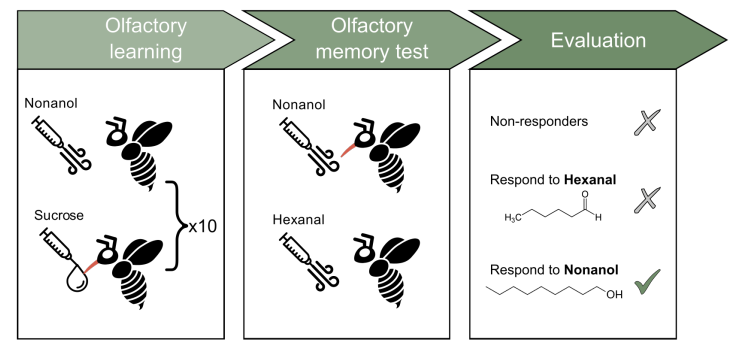

B

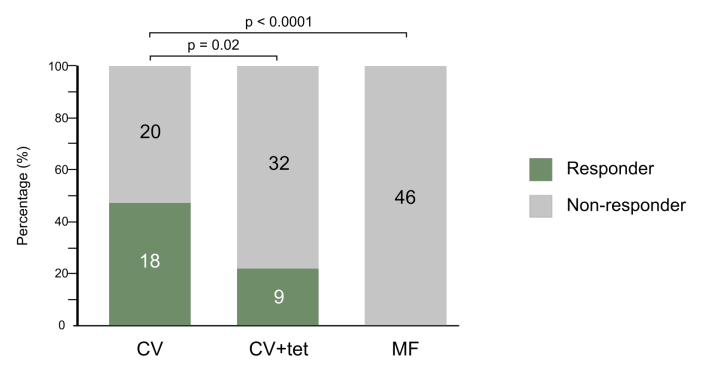

C

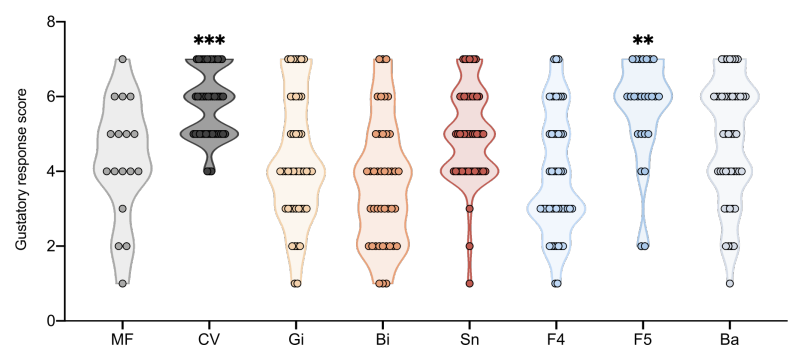

D

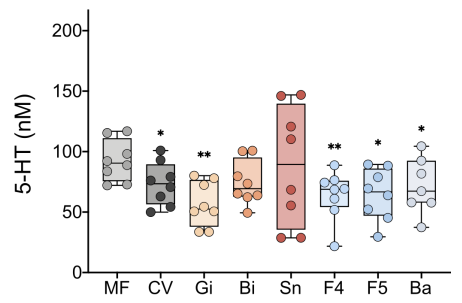

$\mathrm{E}$

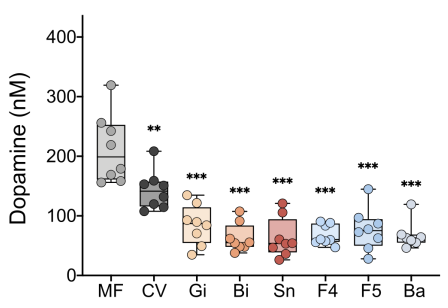

$\mathrm{F}$

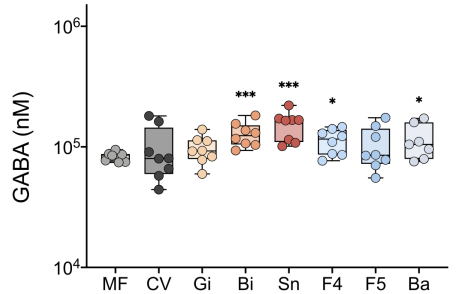

Fig. 1. Gut microbiota alters honeybee behaviors and the concentration of

neurotransmitters in the brain. (a) Olfactory learning and memory test design: 7-day-old (MF) bees were tested. Bees were trained to associate a conditional stimulus (nonanol odor) with a sucrose reward presented in ten successive trials. Bees responded only to nonanol odor in the memory test were considered successful. (b) Ratio of successfully memorized bees in the CV ( $n=38), C V+$ tet $(n=41)$, and MF groups $(n=46)$. Group differences were tested by Chi-squared test. (c) Distribution of gustatory response score of MF ( $\mathrm{n}=17), \mathrm{CV}$

140 bees $(n=46)$, and bees mono-colonized with different core gut bacteria: Gi, Gilliamella

141 apicola $(\mathrm{n}=45) ; \mathrm{Bi}$, Bifidobacterium asteroides $(\mathrm{n}=42) ;$ Sn, Snodgrassella alvi $(\mathrm{n}=50)$;

142 F4, Lactobacillus Firm-4 ( $\mathrm{n}=48)$; F5, Lactobacillus Firm-5 ( $\mathrm{n}=25)$; Ba, Bartonella apis $(\mathrm{n}$ $=46$ ). Each circle indicates a bee response to the provided concentration of sucrose. $* * p<$ 
$1440.01,{ }^{* * *} \mathrm{p}<0.001$ (Mann-Whitney $u$ test for the comparison with the MF group). (d-f)

145 Concentrations of (d) 5-HT, (e) dopamine, and (f) GABA in the brains of MF (n=8), CV (n

$146=8)$, and mono-colonized ( $\mathrm{n}=8$, except $\mathrm{n}=7$ for Ba group) bees. Differences between

147 bacteria-colonized and MF bees were tested by Mann-Whitney $u$ test $\left({ }^{*} \mathrm{p}<0.1,{ }^{* *} \mathrm{p}<0.01\right.$,

$148 \quad * * * p<0.001)$. Error bars represent min and $\max (\mathbf{d}-\mathbf{f})$. 


\section{Transcriptomic and alternative splicing profiles in the brain}

The performance in PER and olfactory learning-memory behavior of honeybees is primarily associated with the gene expression profiles in the brain ${ }^{27}$. In total, our RNA sequencing analysis revealed that 713 genes were differentially expressed in bees colonized with gut members compared to MF bees (Supplementary Data 1), and different bee groups exhibited distinctive brain gene expression profiles (Supplementary Fig. 2a). Insect odorant-binding proteins (OBPs) play key roles in transport odorant molecules to olfactory receptors ${ }^{28}$, which is essential for the detection and distinguishment of specific odors $^{29}$. Here, we found that the G protein-coupled olfactory receptor Or 115 and odorant binding protein Obp14 were both upregulated in the CV group (Fig. 2a, Supplementary microbiota (Fig. 1c). In addition, seven of the ten mrjp family genes of the major royal jelly protein (MRJP) encoding in A. mellifera genome were significantly upregulated in $\mathrm{Bi}$ and Sn groups, while bees colonized with Gilliamella exhibited decreased expression of the mrjp genes (Supplementary Fig. 2b). MRJPs have polyfunctional properties and participate in all major aspects of eusocial behavior in honeybees, such as caste determination and age polyethism ${ }^{30}$. Furthermore, genes encoding vitellogenin and the

166 hexamerin HEX70a, which are both involved in the regulation of bee hormonal dynamics 167 and the transition of foraging behavior ${ }^{31,32}$, were also upregulated in Bi and Sn groups

168 (Supplementary Fig. 2b). The enrichment analysis of differentially expressed genes identified that KEGG pathways including linoleic, alpha-linolenic, arachidonic acids, and glycerophospholipid metabolism were upregulated in brains of different bacteria-

171 colonized groups (Fig. 2b). The glycolysis/gluconeogenesis pathway that is critical for

172 brain physiology via providing the fuel for brain functions ${ }^{33}$ was only upregulated in bees colonized with Gilliamella, while the protein processing, export, and ribosome pathways 
were upregulated in the Sn group. These results showed that the transcriptomic programs are differentially altered in the bacteria-colonized groups.

The gut microbiota does not only regulate gene expression but also affect alternative splicing (AS) of genes in the brain ${ }^{7}$, thus we investigate whether gut bacteria colonized bee brains show different AS events compared with the MF bees. rMATS analysis of the alternative splicing events of the brain genes detected a total of 22,064 events in 5,281 genes, and skipped exon (SE) is the most abundant among different types of AS. About $10-25 \%$ of events for each type of AS showed significantly different inclusion rates in bacteria-colonized bees (Supplementary Fig. 3a). The relative abundance of different types of AS events were similar across bee groups (Supplementary Fig. 3a). However, the UpSet plot shows that the vast majority of events do not intersect between sets, indicating that multiple AS events can occur in a single gene and the gut members cause different AS events (Supplementary Fig. 3b). Interestingly, it has been shown that the gene expression signatures of honeybees with disordered social behaviors are significantly enriched for human autism spectrum disordered (ASD)-related genes ${ }^{34}$. Likewise, the differentially expressed genes in bacteria-colonized bees also overlapped with those from human ASD patients (Supplementary Fig. 3c), implying the involvement of gut microbiota in host behaviors. Besides, dysregulation of alternative splicing in ASD-related genes is also associated with the psychiatric disorder ${ }^{35}$. Thus, we examined the overlap of genes showing significantly differential AS events between MF bees and bacteriacolonized groups with the ASD risk genes from the SPARK for Autism and the SFARI Gene datasets $^{36}$ (Fig. 2c). Three hundred and two of the 2,753 differentially spliced genes

196 in MF bees are associated with human autism, and 64 genes are present in both SPARK 197 and SFARI Gene list (Fig. 2c). Interestingly, almost all identified homologs belong to the high-confidence SFARI gene list (Category 1) that have been clearly implicated in ASD 
199 (Supplementary Data 2). Specifically, we detected differential AS events of genes in MF

200 bees in comparison with bacteria-colonized bees are related to the pathophysiology of

201 ASD. For example, the inclusion rates of both mutually exclusive exon (MXE) and SE

202 events in the Ank2 gene that is important for neuronal migration 37,38 are regulated in $\mathrm{Gi}$,

203 Sn, Bi, F4, and CV groups compared to MF bees (Fig. 2d). The synapse active-zone

204 protein-coding gene Rims 1 with important roles in the maintenance of normal synaptic

205 function $^{39}$ also exhibited different inclusion rates of MXE and SE. Taken together, we

206 identified that the gut microbes not only induce the differential gene expression profiles in

207 the honeybee brain but also mediate AS resulting in specific gene isoforms. Genes

208 essential for bee social behaviors and related to human ASD disease are apparently

209 affected by different gut members, confirming the similarities in genes associated with

210 social responsiveness of humans and honeybees ${ }^{34}$. 
A

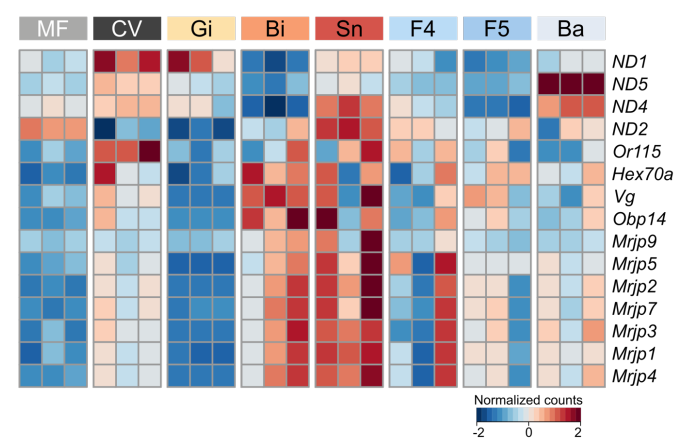

B

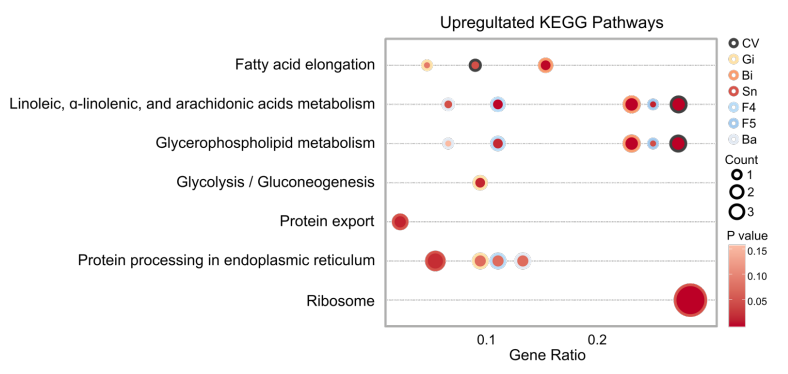

212

213

214

215

216

217

218

219

220
C

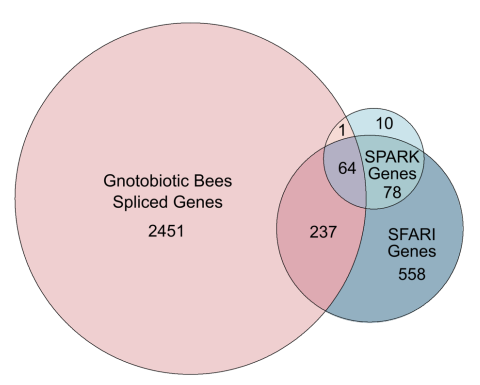

D
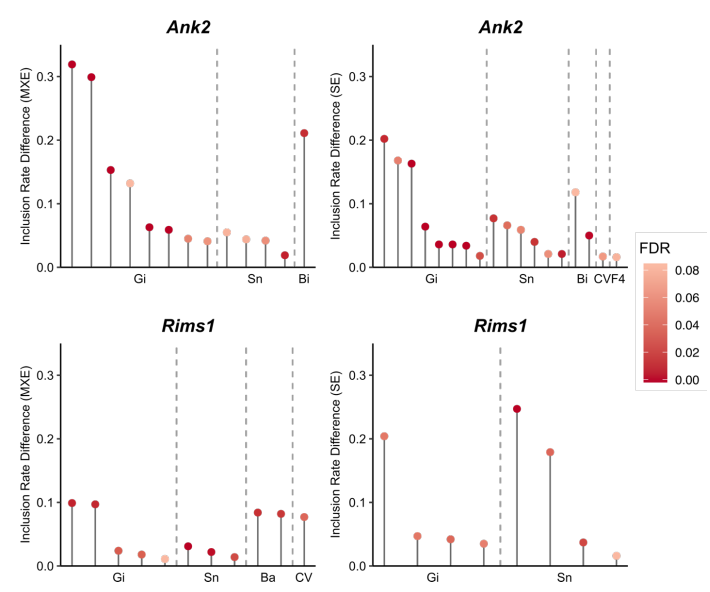

\section{Fig. 2. Gut microbiota impacts gene expression and alternative splicing of high}

confidence ASD genes in the honeybee brain. (a) Heatmap of differentially expressed

genes in the brains of MF and bacteria-colonized bees. Each column represents one brain

sample. Colors indicate the normalized gene counts. (b) KEGG pathways upregulated in the

brains of CV or mono-colonized bees based on the differentially expressed genes. (c) Venn

diagram of differentially spliced genes in the brains between MF and CV/mono-colonized

bees (Gnotobiotic bees spliced genes; $\mathrm{p}<0.05$ ), and their overlap with the SPARK and

SFARI Gene datasets. Differential splicing events were identified by rMATS. (d)

Differentially splicing events (false discovered rate, FDR $<0.1$ ) in Ank2 and Rims 1 present in both SPARK and SFARI Gene datasets. Benjamini-Hochberg corrected p values (FDR)

were calculated by rMATS. MXE, mutually exclusive exon; SE, skipped exon. 


\section{Brain proteomics}

Olfactory learning and memory behaviors of honeybees can be regulated by several proteins in the brain through proteomic analysis ${ }^{40}$. An in-depth proteome profile of the honeybee brain from MF and CV groups identified a total of 3,427 protein counts, 2,845 of which are both detected in MF and CV groups. Three hundred and twenty-two proteins were only found in MF bees, and 257 were exclusive for CV bees (Fig. 3a, Supplementary Data 3). Hierarchical cluster analysis of differentially expressed proteins shared by both 231 groups demonstrated a clear separation between MF and CV bees (Fig. 3b). Notably, the muscarinic acetylcholine receptor ( $\mathrm{mAChR}$ ) involved in the cholinergic neurotransmitter system is upregulated in $\mathrm{CV}$ bees. $\mathrm{mAChR}$ is an acetylcholine binding receptor processing

234 olfactory signals and plays an important role in the retrieval process of associative and 235 non-associative learning and the formation of memory ${ }^{41}$, corroborating our findings of 236 increased memory ability for CV bees (Fig. 1b). We also identified that a splicing factor

237 U2af28 was upregulated in CV brains, supporting the differential patterns of AS in the 238 brain. Interestingly, GO enrichment analysis of unique protein in the CV brain identified

239 that GO terms are related to synaptic neurotransmission and cation/ion transmembrane

240 transportation (Fig. 3c), which are essential for the fundamental functions in the honeybee 241 central nervous system ${ }^{42}$. 
A

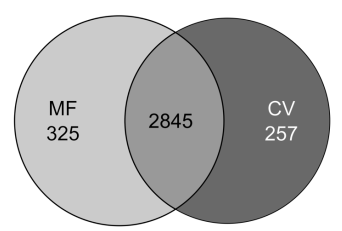

B

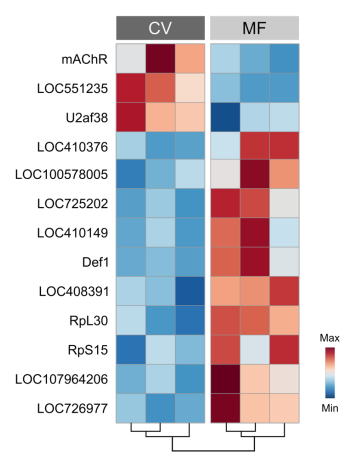

C

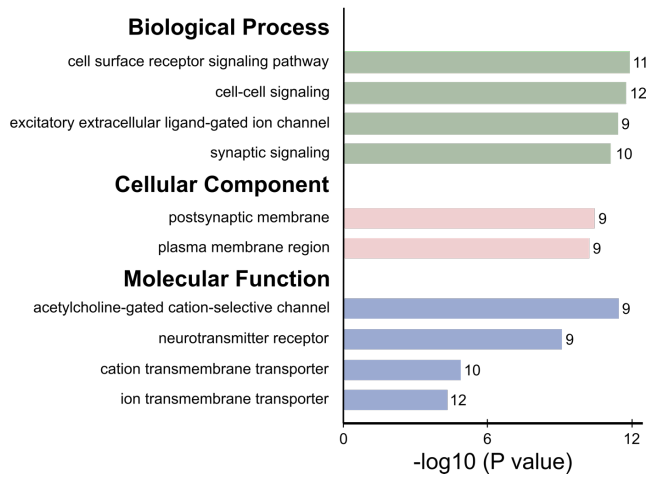

Fig. 3. Proteomic profiling revealed upregulated neurotransmission functions in

conventionalized bee brains. (a) Venn diagrams indicating the numbers of common and unique proteins identified in the brains of $\mathrm{CV}$ and MF bees. (b) Heatmap of differentially expressed proteins in the brains of CV and MF bees. (c) The most significantly enriched GO terms of identified proteins unique to the CV group ( $p<0.0001$, one-way ANOVA test). The number of genes involved in each GO term is displayed on the bars.

We have shown that gene expression, splicing, and neuronal function in the brain are influenced by the gut community, and these can be regulated by small metabolites in the circulatory system ${ }^{6}$. Therefore, we performed quasi-targeted metabolomics analysis of hemolymph samples from gnotobiotic bees. In total, 326 metabolites were identified among bee groups (Supplementary Data 4), and generally, the metabolic signatures of hemolymph samples were significantly different between groups (Fig. 4a, b). Interestingly, GABA and acetylcholine together with several amino acids are the most elevated metabolites in CV hemolymph (Fig. 4a). Lower levels of 5-HT are found in the hemolymph of bees colonized with Gilliamella and the gram-positive gut members, which is consistent with our findings of the effects on the brain neurotransmitter (Fig. 1d). To associate clusters of highly correlated metabolites to particular gut members, we performed the weighted correlation 
262 network analysis (WGCNA) based on the interaction patterns among metabolites, and bees

263 inoculated with different gut microbes were used as the sample trait. WGCNA clustered the

264326 metabolites into eight modules (M) (Supplementary Data 5), in which six modules were

265 significantly correlated with at least one bee group $(\mathrm{p}<0.01$; Fig. $4 \mathrm{c})$. The top two modules,

266 turquoise $\mathrm{M}$ and blue $\mathrm{M}$ were both significantly associated with the CV group (Fig. 4d,

267 Supplementary Fig. 4e), and accordingly, these two modules showed significant correlations

268 between the metabolite significance and the intra-module connectivity for CV bees

269 (Supplementary Fig. 4a, b). The major driving metabolites from the turquoise $\mathrm{M}$ and blue $\mathrm{M}$

270 are involved in the metabolism pathways of amino acid, glycerophospholipid, and

271 carbohydrate (Fig. 4d, Supplementary Fig. 4a, b and e). The black M enriched in amino acid

272 metabolism and some other compounds were the most correlated module to the F4 and F5

273 groups (Fig. 4c, d). Moreover, the Gi group was significantly associated with the red M and

274 pink M, where more metabolites belong to the carbohydrate metabolism pathways (Fig. 4d,

275 Supplementary Fig. 4c-e). This is consistent with the potential of G. apicola for

276 carbohydrate metabolism in the gut ${ }^{16,43}$. 
A

B
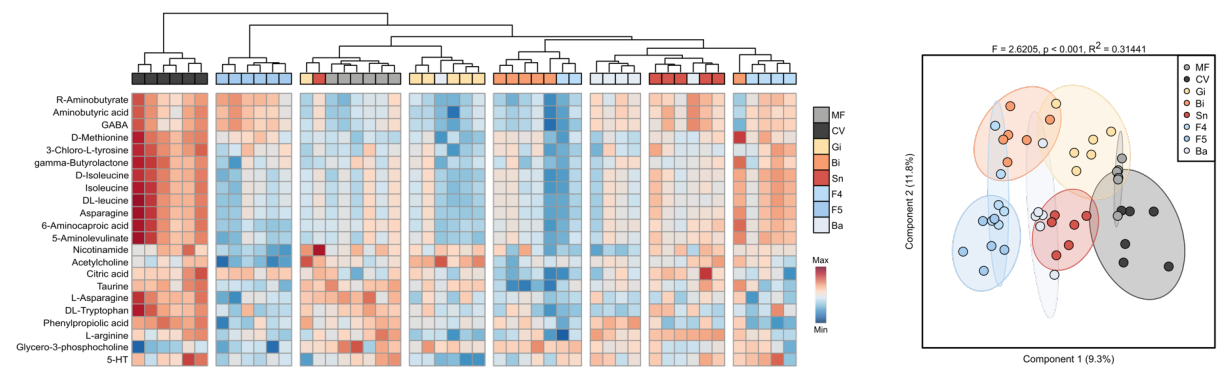

C
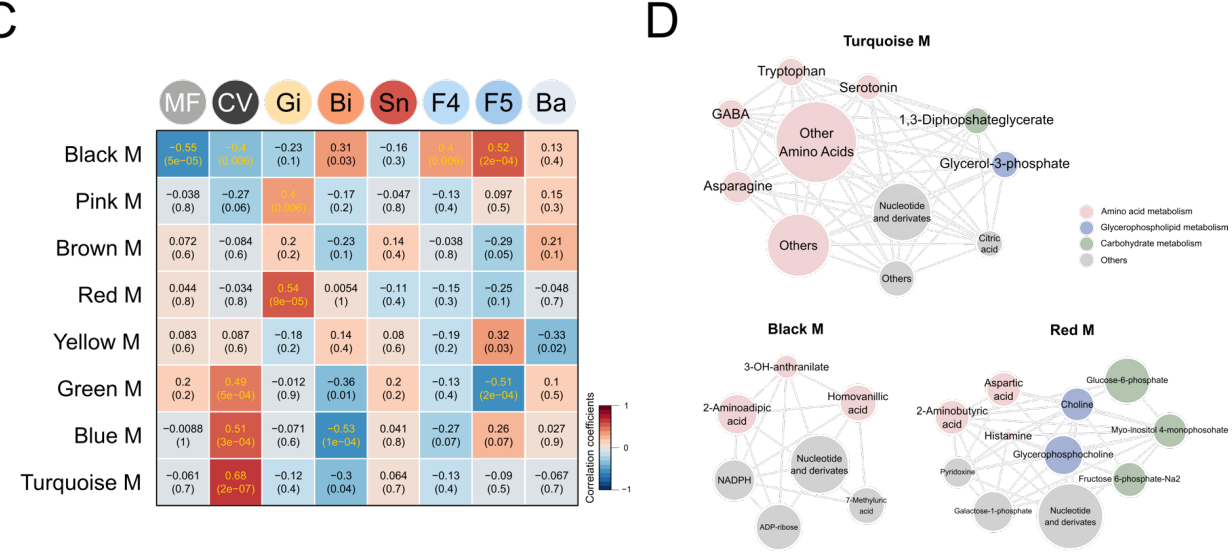

Fig. 4. Hemolymph metabolome influenced by different honeybee gut community

members. (a) Unsupervised hierarchical clustering heatmap of the 22 metabolites that

contribute most to the separation of different groups in hemolymph samples. (b) Sparse

PLS-DA based on all metabolites detected in the hemolymph of bees. Group differences

were tested by PERMANOVA. (c) Weighted correlation network analysis identified eight

coefficients and $p$ values are both shown within the squares (yellow font indicates $p<0.01$ ).

(d) Network diagrams of differential metabolites in the turquoise, black, and red modules

that are significantly correlated to CV, F4, F5, and Gi groups. Circle colors indicate different the metabolites in the modules. 
Given the evidence that the altered metabolites were largely involved in the metabolic network of neurotransmitters, we analyzed the differential level of metabolites focusing on the neurotransmitter metabolic process. Tryptophan (Trp) metabolism mainly controlled by microbiota follows three major pathways in the gastrointestinal tract: the kynurenine (Kyn) pathway, the 5-HT production pathway, and the transformation of Trp into indole by gut microbiota ${ }^{44}$. It showed that 3-indoleacrylic acid (IA), a key component for intestinal homeostasis $^{45}$, was significantly elevated in the hemolymph of CV and F5 groups. While KA was not affected, Kyn was reduced in CV bees (Fig. 5). The glutamate metabolism pathway was mostly regulated in $\mathrm{CV}, \mathrm{Bi}, \mathrm{Sn}$, and F5 groups (Fig. 5). Although glutamine was only increased in $\mathrm{CV}$ bees, GABA was upregulated in the hemolymph of $\mathrm{CV}, \mathrm{Sn}$, and $\mathrm{F} 5$ bees,

300 which agrees with the elevated level of GABA in the brains (Fig. 1f). In addition,

301 glycerophospholipid metabolism also plays an important role in maintaining positive mental

302 health $^{46}$. The hemolymph metabolites in glycerophospholipid metabolism were regulated in

303 the CV, Gi, Sn, F5, and Ba groups compared to the MF group (Fig. 5). Specifically,

304 acetylcholine associated with the olfactory learning and memory ability in honeybee was 305 upregulated in the presence of Gilliamella (Fig. 5), coinciding with the increased level of

$306 \mathrm{mAChR}$ in the brain (Fig. 3b). These results indicate that neurotransmitter related

307 metabolisms are regulated by distinctive gut members, which may be a key mechanism of gut microbiota in modulating the brain functions. 

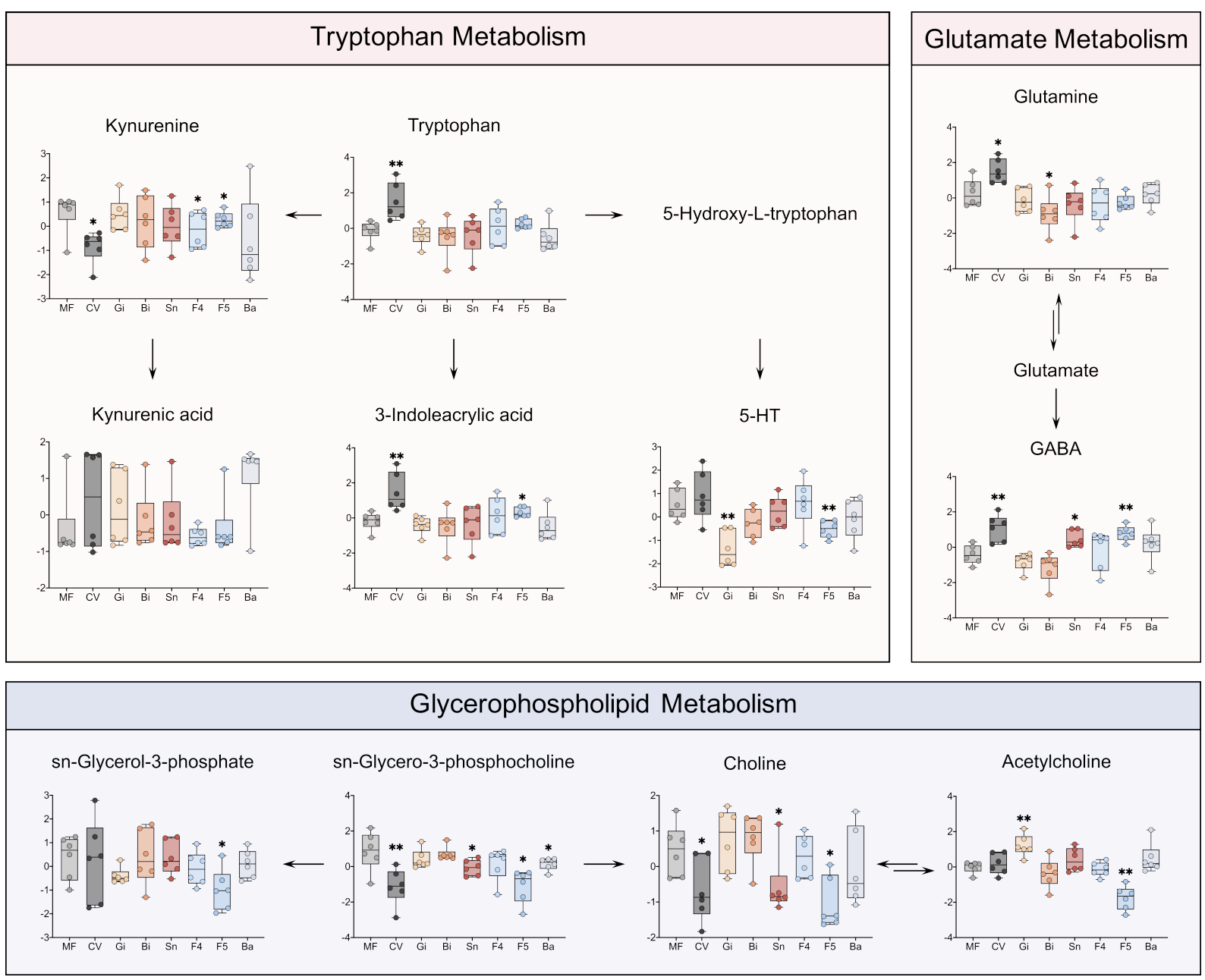

Fig. 5. Disturbance of amino acid and glycerophospholipid metabolism pathways in

311 honeybees colonized with different gut community members. Each dot represents the

312 normalized concentration of differential hemolymph metabolite mapped into the tryptophan,

313 glutamate, and glycerophospholipid metabolism pathways. Differences between MF and the

314 other groups were tested by Mann-Whitney $u$ test $\left({ }^{*} \mathrm{p}<0.1,{ }^{*} \mathrm{p}<0.01\right)$. Error bars represent min and max. 
Antibiotic treatment disturbs social behavior via regulating brain transcription and

amino acid metabolisms

So far, our results showed that the colonization of different gut bacteria affects the honeybee behaviors under lab conditions, which is associated with the altered brain gene expression profiles, as well as the circulating and brain metabolism. We next wondered whether the perturbation of gut microbiota disturbs bee behaviors under field condition. Newly emerged bees were labeled with color tags and were then introduced to new hives with laying queens. After being returned to the hives for one week, colonies were fed wild honey or tetracycline suspended in wild honey for 5 days (Fig. 6a). The number of capped brood cells were counted, and post-treatment survival was assessed by counting the number of remaining marked bees. Although there is an increasing number of capped brood cells in the control hives, not a single capped brood was observed in the treatment group on Day 17, 18, and 19 (Fig. 6c). However, the number of recovered bees was not significantly different between control and treatment groups either before (Day 6) or after (Day 13 and 19) antibiotic treatment (Fig. 6d). Further, developing eggs and larvae were present in the brood cells of control hives with royal jelly replenished in the bottom, while only few eggs were observed in the treatment group without hatching during the whole experiment period (Fig. $6 b)$. All these results indicate that antibiotic treatment did not obviously decrease the total number of adult bees in the hives, but disrupt the behaviors of bees without brood care. Moreover, antibiotic treatment impacts the gut appearance that the rectums of control groups are full of yellow pollens, while those of treated nursing bees were more translucent, suggesting a malnutrition status (Fig. 6b). We further characterized the composition of the gut community at both phylotype- and SDP-level through metagenomic sequencing.

339 Although the gut community composition displayed no significant difference at pretreatment sampling points, they displayed changes in treated bees and recovery for 7 days 
341 (Fig. 6e, Supplementary Fig. 5a). Specifically, the treatment group had a higher fraction of

342 Gilliamella, while the relative abundance of Bifidobacterium was reduced. Tetracycline

343 treatment affected the SDP-level profiles, and the relative abundances of Bifido-1.1, Bifido-

344 1.2, Firm5-2, and Firm5-3 were reduced in antibiotic-treated samples (Supplementary Fig.

$345 \quad 5 b-e)$.

Consistent with the findings for gnotobiotic bees, we also identified a differential

347 profile of brain gene expression for antibiotic-treated bees. The most altered genes belong to

348 the MRJP, vitellogenin, odorant binding protein, and olfactory receptor families (Fig. 6f).

349 Remarkably, Gr10 and ACSF2 that are both primarily associated with the nursing and brood-

350 care behavior were significantly reduced by the treatment ${ }^{47,48}$. Additionally, we detected the

351 differentially abundant metabolites in the gut contents of antibiotic-treated bees from the

352 field experiment. The metabolomic profiles are clearly distinct between the two groups, and

35354 metabolites were significantly different in the gut of treated bees (Fig. $6 g, \mathrm{~h}$ ).

354 Intriguingly, 5-AV, a $\mathrm{GABA}_{\mathrm{A}}$ receptor agonist affecting inhibitory GABA signaling ${ }^{49}$, is the 355 most elevated compound in control bees (Fig. 6h, i). Conversely, Kyn is enriched in the gut

356 of antibiotic-treated bees (Fig. 6h). The Kyn/Trp ratio was increased in the treatment group,

357 while the ratio of KA/Kyn and the level of IA were decreased (Fig. 6i-k), which confirms the

358 roles of gut microbiota in the tryptophan metabolism shift (Fig. 5). These results confirm our

359 hypothesis that the gut microbiota affects honeybee behaviors under field conditions via the

360 regulation of gut metabolism and gene expression in the brain. 
A

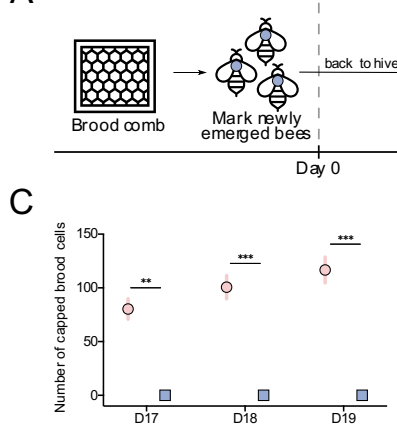

$\mathrm{E}$

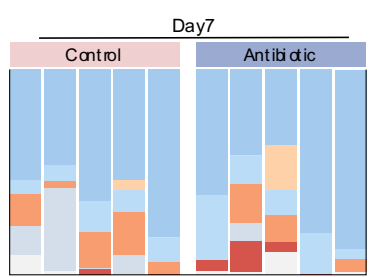

B

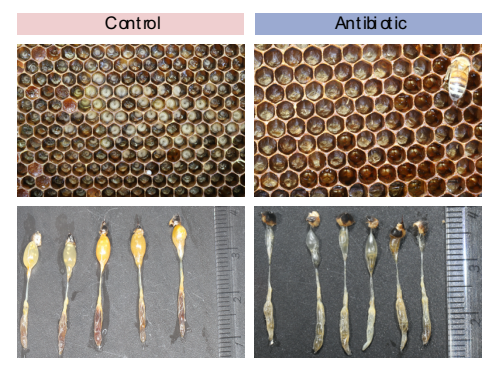

$\mathrm{F}$
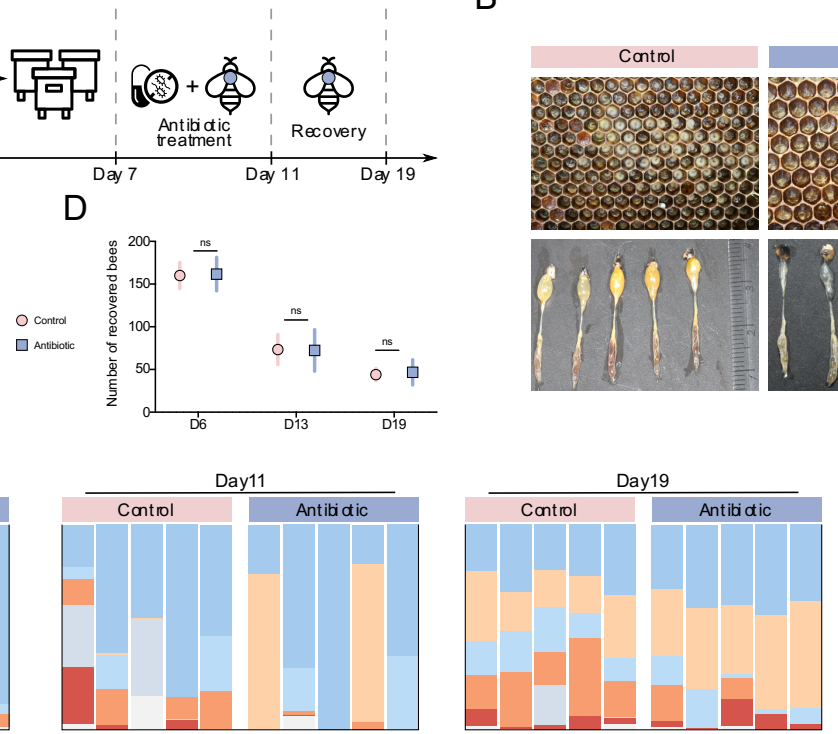

$\square$ Gilliamella

$\square$ Bifidobacterium

$\square$ Snodgrassella

$\square$ Lactobacillus Firm-4

$\square$ Lactobacillus Firm-5 $\square$ Frischella

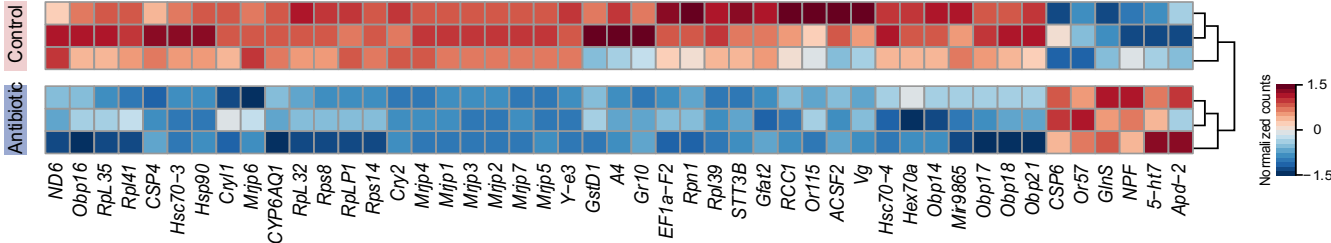

G

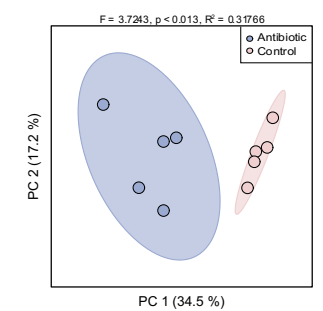

$\mathrm{H}$

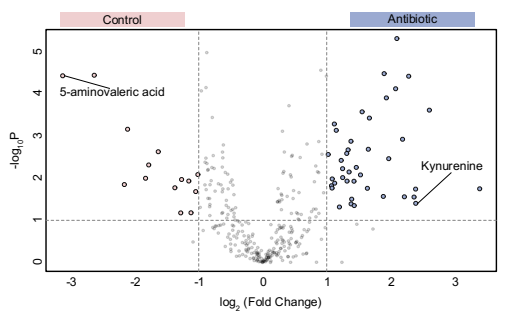

I

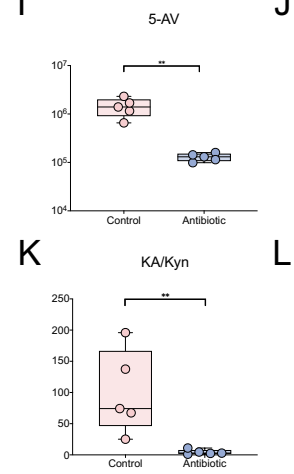

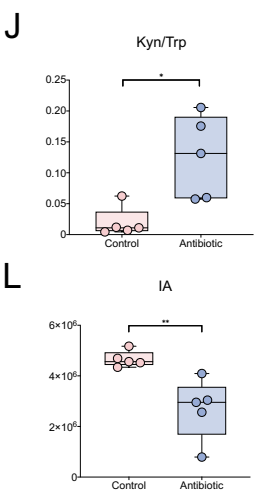

Fig. 6. Antibiotic treatment affects honeybee social behaviors via regulating brain

transcription and amino acid metabolisms. (a) Schematic of field experiments design for

364 honeybee behavior. Age-controlled bees were treated with tetracycline for 5 days (Day 7-

365 11) in the hive and recovered for 7 days (Day 11-19). (b) Images of brood frames and dissected guts of control and antibiotic-treated groups. (c) Number of capped brood cells

367 during the recovery stage (Day 17, 18, and 19) in three independent colonies of control and antibiotic-treated group, respectively. (d) The number of labelled workers recovered from 
369 the hive on Day 6 and 13 in three colonies of each group. Differences between antibiotic-

370 treated bees and the control group were tested by multiple two-tailed $t$ test with Benjamini-

371 Hochberg correction $(* \mathrm{FDR}<0.05, * * \mathrm{FDR}<0.01, * * * \mathrm{FDR}<0.001)$. (e) Relative

372 abundance of phylotypes in metagenomic samples from control and antibiotic-treated groups

373 before the antibiotic treatment (Day 7), at post-treatment (Day 11), and one week after the

374 recovery (Day 19). (f) Heatmap of differentially expressed genes in the brains between

375 control and antibiotic-treated bees. (g) Principal coordinate analysis based on all metabolites

376 detected in guts of control and antibiotic-treated bees. Group differences were tested by

377 PERMANOVA. (h) Volcano plot showing the differentially regulated metabolites.

378 Metabolites significantly enriched in control bees are shown in pink, and those enriched in

379 antibiotic-treated bees are in blue. (i-l) Boxplots of (i) the kynurenine (Kyn)/tryptophan

380 (Trp) ratio, (j) the kynurenic acid (KA)/kynurenine (Kyn) ratio, the concentration of (k) 3-

381 indoleacrylic acid (IA), and (I) 5-aminovaleric acid (5-AV) in the guts of control and

382 antibiotic-treated bees. Group differences were tested by Mann-Whitney $u$ test $\left({ }^{*} \mathrm{p}<0.1,{ }^{* *} \mathrm{p}\right.$

$383<0.01)$. Data are shown as mean \pm SEM (c-d). Error bars represent min and max (i-l). 


\section{Discussion}

Honeybees are eusocial insects that exhibit complex social, communication, and navigational behaviors with rich cognitive repertoire, such as color vision, pattern recognition, as well as learning and memory ${ }^{50}$. Within the colony, honeybees are characterized by the division of labor, showing striking behavioral and physiological differences between castes ${ }^{51}$. Although the gut microbiota composition is mostly conserved in worker bees, it differs in individuals with different behavior and physiology, such as caste, age, and worker task ${ }^{52,53}$, which suggests that the gut microbiota might be involved in the behavior of honeybees. While our previous study shows that bee gut microbiota alters the olfactory sensitivity ${ }^{14}$, the impact of microbiota on more behavioral symptoms has not been described. We report herein that a conventional gut microbiota is required for the learning ability and the establishment of memory. Although the olfactory associative learning of honeybees is largely dependent on the microbiota, the effect of gut bacteria on modulating bumblebee's visual learning and memory is not clear ${ }^{54}$, suggesting that the mechanism underpinning the gut-brain interactions differs for social bees, or for olfactory and visual processing. Notably, we performed the associative appetitive learning assay in this study,

400 while the aversive learning is also pivotal for bees to escape and avoid predators and 401 pesticides $^{55}$. Appetitive and aversive olfactory learning are mediated by relatively independent neural systems. Dopamine is crucial for aversive learning ${ }^{56}$, which is also regulated by the gut microbiota (Fig. 1e). Further evaluation of microbiota on different

404 behaviors would assist to fully understand the mechanism of gut-brain interaction.

Olfactory and the ability of learning and memory is crucial for honeybees to cope with 406 individual and social tasks, such as feeding and foraging ${ }^{57}$. Our hive experiments 
capped brood was observed in antibiotic-treated hives, suggesting a significant role of the normal gut microbiota in honeybee behaviors within the colony. The number of capped brood cells is a measure of the colony strength, which could also be influenced by the status

411 of the egg-laying queen and the colony population size ${ }^{58}$. However, the total number of individual bees was not obviously reduced, and newly laid eggs were continuously observed in the treatment hives, implying that the perturbation of gut microbiota affects the normal

414 hive behaviors of nurse bees and the colony reproduction.

By generating single bacterial associations, we intended to dissect the individual and combined effects of each core gut member in the sugar sensitivity. However, it showed that only conventionalized bees had a higher sensitivity, and individual gut members were not sufficient to improve the PER score, suggesting an integrative effect of the gut members, which is also reported for the Drosophila microbiota on the host learning ${ }^{59}$. Under field conditions, antibiotic treatment did not completely eliminate any core gut species but perturbed the relative abundance of the SDPs of Lactobacillus Firm-5 and Bifidobacterium, which indicates that the normal microbial community structure is required for the colony health. It has been shown that antibiotic exposure impacts bee health and dramatically reduced the survival rate with dysbiosis on the relative abundance of different bacterial genera and the fine-scale genetic diversity of the gut community ${ }^{60}$. In addition to the increased susceptibility to ubiquitous opportunistic pathogens ${ }^{61}$, the colony losses resulting from antibiotic treatment could be partly due to the altered hive behaviors.

Our RNA-seq analysis of gnotobiotic bee brains showed that numerous transcripts differed in expression levels, moreover, genes related to honeybee labor division and olfactory ability were altered by gut bacteria. For example, genes encoding the MRJPs

431 involved in the learning and memory abilities of honeybees were upregulated in bacteria432 colonized bees. Consistently, it has been reported that the expression level of mrjpl and 
mrjp 4 are repressed in the brains of imidacloprid treated bees, which also exhibit impaired learning ${ }^{27}$. The expression level of $V g$ was disturbed by gut microbiota in both laboratory experiments and in the hive, corroborating with the previous finding in MF bee abdomen ${ }^{14}$. Vitellogenin is a nutritional status regulator that influences honeybee social organization and stress resilience ${ }^{62}$. The expression of $V g$ and survivorship could be elevated by the addition of pollen to the diet ${ }^{63}$, whereas the effect is alleviated by the disturbance of gut community ${ }^{64}$, indicating the important role of gut microbiota in $\mathrm{Vg}$ regulation via the nutritional metabolism.

Homologous molecular mechanism in social responsiveness has been documented between honeybee and human ${ }^{34}$. The transcription profile in brains of bees with disordered social behaviors is distinct from that of normal bees, and differently expressed genes in unresponsive individuals are enriched for human ASD-related genes. These genes are also found associated with the polymorphism of the halictid bee Lasioglossum albipes, indicating their implications in social behaviors ${ }^{65}$. Despite the disturbed gene expression level, AS patterns of the ASD-related genes are also highly correlated to mental disorders ${ }^{7}$. In our dataset, the analysis of gene splicing identified extensive differences among bacteriacolonized groups of bees, and the altered genes compared to MF bees largely overlapped with the SPARK and SFARI gene datasets for Autism (Fig. 2). The MXE and SE events of two high-confidence ASD risk genes, Ank2 and Rims1, were predominantly affected by the gut bacteria. These two genes are also found affected in the brains of mice colonized by ASD-human gut microbiota ${ }^{7}$. Correspondingly, our brain proteomics revealed that the splicing factor was upregulated in CV brains, supporting the contribution of microbiota to splicing regulation. All these findings demonstrate a deep conservation for genes related to social responsiveness of human and distantly related insect species, and reflect a common role of the gut microbes implicated in the evolution of sociality ${ }^{66}$. 
Neurotransmitters that carry and pass information between neurons are essential for brain functions, which are important modulators of behaviors. In honeybees, four monoamine neurotransmitters play important roles in learning and memory ${ }^{22}$, and olfactory sensitivity ${ }^{22,23}$. In addition, GABA and acetylcholine have been physiologically characterized to induce currents between neurons within the olfactory pathways and contribute to the odor memory formation ${ }^{42}$. Concentrations of most identified neurotransmitters were regulated by different gut members, corroborating with the roles of gut microbiota in the altered behaviors in the lab and hive experiments. Alternatively, it recently shows that nestmate recognition cues are defined by gut bacteria, possibly by cuticular hydrocarbon ${ }^{67}$. In leaf-cutting ants and termites, gut microbiota suppression by antibiotics also influences the recognition behavior toward nestmates, which may be directed by the bacterial metabolites as recognition cues in the feces ${ }^{68,69}$. Nevertheless, the effect of gut community is mainly driven by the microbial metabolism, specifically the amino acid and lipid metabolic pathways, which can further influence the circulation system and the synthesis of neuroactive molecules of the host. Perturbation in gut tryptophan metabolism

474 has been associated with neuropsychiatric disorders in human and Drosophila model, 475 characterized by reduced plasma level of tryptophan ${ }^{70}$, high IDO1 activity ${ }^{71}$, and high level of 5-HT in brains ${ }^{72}$. In honeybees, 5-HT was also elevated in MF bee brains compared to bacteria-colonized counterparts, moreover, the level of IDO1 activity (assessed by Kyn/Trp ratio) was higher in the gut of antibiotic-treated bees in the field colony. In addition, acetylcholine synthesized in the glycerophospholipid pathway is a neurotransmitter crucial 480 for the olfactory learning and memory ability in honeybees. Our results revealed that gut microbiota mediated the cholinergic metabolism in the hemolymph, and correspondingly, the brain proteomics showed an increased level of the muscarinic acetylcholine receptor in 
484 learning and foraging behaviors ${ }^{41}$. Moreover, the stimulation of the mAChR of honeybee

485 increases the volume of the mushroom body neuropil, which mimics the reinforcement of

486 cholinergic neurotransmission in foraging bees ${ }^{73}$. A reduced mushroom body calycal growth

487 is also associated with lower learning performance in bumblebees through micro-computed

488 tomography scanning ${ }^{74}$. It would be interesting to investigate whether gut microbes impact

489 the structural changes of the brain in future studies.

It is increasingly realized that gut microorganisms may influence the development of

491 social behaviors across diverse animal hosts ${ }^{66}$. While hypothesis-generating, translating

492 these correlations into actionable outcomes is challenging in humans. Honeybees are

493 colonial and highly social with multiple symbolic behaviors, which offer an experimental

494 tool to investigate the relationship between the microbiota and host brain functions and help

495 to uncover the causal mechanisms underlying sociability. Our study highlights multiple

496 parallels between honeybee and human that gut microbiota plays an important role in host

497 brain functions. The development of genetic tools manipulating both the bee host and the gut

498 bacteria would facilitate the investigation of the molecular basis of host-microbe interactions

499 via the gut-brain axis ${ }^{75,76}$. 


\section{Generation of microbiota-free, mono-colonized and conventionalized honeybees}

Microbiota-free (MF) bees were obtained as described by Zheng et al. ${ }^{16}$ with modifications.

503 Late-stage pupae were removed manually from brood frames and placed in sterile plastic bins. The pupae emerged in an incubator at $35^{\circ} \mathrm{C}$, with humidity of $50 \%$. Newly emerged MF bees (Day 0) were kept in axenic cup cages with sterilized sucrose syrup (50\%, wt/vol) for $24 \mathrm{~h}$ and divided into three groups: 1) MF, 2) mono-colonized (MC) and 3) conventional (CV) bees. For each setup, 20-25 MF bees (Day 1) were placed into one cup cage, and the bees were feeding on the corresponding solutions or suspensions for $24 \mathrm{~h}$. For the MF group, $1 \mathrm{~mL}$ of $1 \times$ PBS was mixed with $1 \mathrm{~mL}$ of sterilized sucrose solution $(50 \%$, wt $/ \mathrm{vol})$ and $0.3 \mathrm{~g}$ sterilized pollen. For the MC group, stocks of Gilliamella apicola (W8127), Snodgrassella alvi (W6238G3), Bifidoobacterium asteroides (W8113), Bartonella apis (B10834G6), Lactobacillus sp. Firm-4 (W8089), and Lactobacillus sp. Firm-5 (W8172) in 25\% glycerol stock at $-80^{\circ} \mathrm{C}$ were resuspended in $1 \mathrm{~mL} 1 \times \mathrm{PBS}$ (Solarbio, Beijing, China) at a final $\mathrm{OD}_{600 \mathrm{~nm}}$ of 1 , and then mixed with $1 \mathrm{~mL}$ sterilized sucrose solution $(50 \%$, wt/vol) and $0.3 \mathrm{~g}$

516 bees from their hives of origin were mixed with $1 \mathrm{~mL} 1 \times \mathrm{PBS}, 1 \mathrm{~mL}$ sterilized sucrose

517 solution $(50 \%$, wt/vol) and $0.3 \mathrm{~g}$ sterilized pollen. Then MF, MC, and CV bees were

518 provided sterilized sucrose $(0.5 \mathrm{M})$ with sterile pollens and kept in an incubator $\left(35^{\circ} \mathrm{C}, \mathrm{RH}\right.$

$51950 \%$ ) until day 7. Brains, guts, and hemolymph of bees were collected on day 7 for further 520 analysis. 
Colonization levels of MF and MC bees were determined by colony-forming units from dissected guts, as described by Kwong et al. ${ }^{77}$. Colonization levels of CV bees were determined by quantitative PCR as previously described by Engel et al. ${ }^{13}$. All qPCR reactions were carried out in a 96-well plate on the StepOnePlus Real-Time PCR system (Applied Biosystems; Bedford, MA, USA) with the thermal cycling conditions as follows: denaturation stage at $50^{\circ} \mathrm{C}$ for $2 \mathrm{~min}$ followed by $95^{\circ} \mathrm{C}$ for $2 \mathrm{~min}, 40$ amplification cycles at $95^{\circ} \mathrm{C}$ for $15 \mathrm{~s}$, and $60^{\circ} \mathrm{C}$ for $1 \mathrm{~min}$. Melting curves were generated after each run $\left(95^{\circ} \mathrm{C}\right.$ for $15 \mathrm{~s}, 60^{\circ} \mathrm{C}$ for $20 \mathrm{~s}$ and increments of $0.3^{\circ} \mathrm{C}$ until reaching $95^{\circ} \mathrm{C}$ for $15 \mathrm{~s}$ ) to compare dissociation characteristics of the PCR products obtained from gut samples and positive $\mu \mathrm{l}\left(0.2 \mu \mathrm{M}\right.$ of each forward and reverse primer; and 1x SYBR ${ }^{\circledR}$ Select Master Mix, Applied Biosystems; Bedford, MA, USA) with $1 \mu$ of DNA or cDNA (to assess virus loads). Each

534 plate contained a positive control and a water control. After the calculation of the bacterial 16S rRNA gene copies, normalization with the actin gene was carried out to reduce the effect of gut size variation and extraction efficiency. In brief, bacterial 16S rRNA gene copies were normalized to the medium number of actin gene copies by dividing by the 'raw' copy number of actin for the given sample and multiplying by the median number of actin

539 gene copies across all samples. Universal bacteria primers (Forward: 5' AGGATTAGATACCCTGGTAGTCC-3', Reverse: 5'- YCGTACTCCCCAGGCGG-3') ${ }^{13}$ and Apis mellifera actin (Forward: 5' - TGCCAACACTGTCCTTTCTG -3', Reverse: 5'AGAATTGACCCACCAATCCA -3' $)^{78}$ were used here.

\section{Tissue collection}

544 The whole guts were dissected by tweezers disinfected with $75 \%$ alcohol. Dissected guts 545 were directly crushed in $25 \%$ ( vol/vol) glycerol on ice for bacterial load quantification or 546 collected into an empty $1.5-\mathrm{mL}$ centrifuge tube for metagenomic sequencing and 
547 metabolomics analysis. All gut samples were frozen at $-80^{\circ} \mathrm{C}$ until analysis. Honeybee

548 brains were collected using a dissecting microscope (Canon). Individual bee was fixed on

549 beeswax using two insect needles through the thorax. After removing the head cuticle, the

550 whole brain was placed on a glass slide and soaked in RNAlater (Thermo; Waltham, MA,

551 USA) or proteinase inhibitor (Roche; Mannheim, Germany) for gene expression profiling,

552 proteome analysis, and neurotransmitters concentration quantification. Then hypopharygeal

553 glands, salivary glands, three simple eyes, and two compound eyes were carefully removed.

554 Dissected brains were kept frozen in $-80^{\circ} \mathrm{C}$. Hemolymph was collected using a $10 \mu \mathrm{L}$

555 pipettor (Eppendorf; Hamburg, Germany) from the incision above the median ocellus. A

556 minimum of $50 \mu \mathrm{L}$ of hemolymph was collected from 10 bees into a $1.5-\mathrm{mL}$ centrifuge tube.

557 During the collection process, tubes are temporarily preserved on dry ice and subsequently

558 stored at $-80^{\circ} \mathrm{C}$ until analysis.

\section{In laboratory honeybee behavior experiment}

\section{$560 \quad$ Learning and memory}

561 We measured the olfactory learning and memory ability of seven-day-old MF, CV, and

$562 \mathrm{CV}+$ tet bees. MF and CV bees were generated as described above. $\mathrm{CV}+$ tet bees were fed 450

$563 \mu \mathrm{g} / \mathrm{ml}$ (final concentration) of tetracycline suspended in sterilized 0.5 M sucrose syrup on Day

5645 after the eclosion for $24 \mathrm{~h}$ and then were fed sucrose syrup for another $24 \mathrm{~h}$ for recovery.

565 Experiments of olfactory learning and memory were performed as previously described ${ }^{20,79}$

566 with modifications (Fig. 1a). In brief, bees were starved for $2 \mathrm{~h}$ by removing sugar syrup and

567 bee bread from the cup cage before the test and were then mounted to modified $0.8 \mathrm{~mm}$ wide

568 bullet shell with sticky tape restraining harnesses (Supplementary Movie 1). The whole

569 experiment was performed in a room with a stable light source at room temperature. Each bee

570 individual was checked for their intact proboscis extension response by touching the antennae 
with 50\% sucrose solution without subsequent feeding $15 \mathrm{~min}$ before the experiment.

572 Nonanol (olfactory learning; Sigma-Aldrich; Saint Louis, MO, USA) and hexanal (negative

573 control; Macklin; Shanghai, China), which could be distinguished by honeybee ${ }^{80}$, were used inter-trial interval of 10 min to associate nonanol odor as conditioned stimulus with a reward of $50 \%$ sucrose solution as unconditioned stimulus. delivered to the bee using a syringe needle, which directly touched the proboscis to evoke PER. Once the 10 trials of a conditioning session were completed, bees were kept in the dark without being fed for $3 \mathrm{~h}$. Two unreinforced olfactory memory tests were administered $3 \mathrm{~h}$ after olfactory conditioning: one with the conditioned stimulus odor (nonanol) and one with a novel odor (hexanal). The order of presentation was randomized across subjects. A clean and tasteless injector was delivered to the bee after each odor test to exclude visual memory

589 of reward during olfactory conditioning. Bees only extending the proboscis to nonanol odor were considered as successful memorized individuals (Fig. 1a).

\section{Gustatory responsiveness}


from the cup cage. Bees were then mounted to modified $2.0-\mathrm{mL}$ centrifuge tubes using

Parafilm M (Bemis; Sheboygan Falls, WI, USA), and they could only move their heads and propodeum for antennae sanitation. Individual responsiveness was measured by presenting a series concentration of sucrose solutions $(0,0.1,0.3,1,3,10$, and $30 \%$; wt/vol $)$ to the antennae of bees ${ }^{81}$. Before each sucrose solution presentation, all bees were tested for their response to pure water in order to control for the potential effects of repeated sucrose stimulations that may lead to either sensitization or habituation ${ }^{82}$. The inter-stimulus interval between water and sucrose solution was $4 \mathrm{~min}$. When a bee's antenna is stimulated with a sucrose solution of sufficient concentration, the bee reflexively extends its proboscis. The lowest sucrose concentration at which an individual responded by extending its proboscis was recorded and interpreted as its sugar response threshold. At the end of the experiment, a gustatory response score was obtained for each bee, which is based on the number of sucrose concentrations to which the bees responded. The response was arbitrarily quantified with scores from 1 to 7 , where 1 represented a bee that only responded to the highest sucrose

611 'failed' response was considered to be an error and the bee was deemed to have responded to

612 that concentration as well. Bees that did not respond to any of the sucrose concentrations were excluded from further analyses. In addition, bees that responded to all concentrations of

614 sucrose solutions and all presentations of water were also excluded as they appeared not to 615 be able to discriminate between sucrose and water ${ }^{82}$.

\section{Hive behavior experiment}


independent single-cohort colonies were set up as previously described ${ }^{83}$. Briefly, brood frames were collected from a single hive and adult bees were brushed off. The frames were then kept in the laboratory incubating at $35^{\circ} \mathrm{C}$ and $50 \%$ relative humidity. In two days, about 1,000 bees emerged from each frame in the incubator, and we labeled 300 individuals with colored tags on their thorax. All newly emerged bees were then introduced to new empty hives together with a newly mated laying queen ${ }^{84}$. Two hives for control and treatment were established. Control colony bees were fed wild honey along with the whole experiment, and treatment groups were fed wild honey suspended with $450 \mathrm{ug} / \mathrm{ml}$ of tetracycline (final concentration) from Day 7 after the establishment of hives (Fig. 6a), and the antibiotic treatment lasted for 5 days. The number of capped brood cells was counted every day, and post-treatment survival in the hive was assessed by counting the number of remaining marked bees of the whole hive ${ }^{61}$. Marked bees for both control and treatment groups were collected from each hive at time points of 7, 11, and 19 day following the set-up of hives, and the hind guts and brain tissue were dissected. All samples were stored at $-80^{\circ} \mathrm{C}$ until analysis.

\section{Gut DNA extraction and metagenomic sequencing}

Bee individuals of either control or antibiotic groups were sampled on day 7, 11, and 19 during the hive behavior experiment (Fig. 6a). Total genomic DNA of the gut microbiota was extracted from the whole gut homogenate using CTAB method as previously described $^{14}$. DNA samples were sent to Novogene Bioinformatics Technology Co. Ltd. (Beijing, China) for shotgun metagenome sequencing. Sequencing libraries were generated using NEBNext Ultra ${ }^{\mathrm{TM}}$ II DNA Library Prep Kit for Illumina (New England Biolabs; Ipswich, MA, USA), and the library quality was assessed on Qubit 3.0 Fluorometer (Life Technologies; Grand Island, NY, USA) and Agilent 4200 (Agilent, Santa Clara, CA) system. 
Diego, CA, USA) and 150 bp paired-end reads were generated. The SDP- and phylotypelevel community structure of each metagenomic sample was profiled following the Metagenomic Intra-Species Diversity Analysis System (MIDAS) pipeline ${ }^{85}$. A custom bee gut bacteria genomic database was generated based on 407 bacterial isolates from honeybees and bumblebees (Supplementary Data 6). Before the classification, we removed reads belonging to the honeybee reference genome (version Amel_HAv3.1) using KneadData v 0.7.3. We then ran the 'species' module of the 'run_midas.py' and 'merge_midas.py' scripts in MIDAS with our custom bacterial genome database, which aligned reads to universal single-copy gene families of phylogenetic marker genes using HS-BLASTN to estimate the abundance of phylotypes and SDPs for each sample. Local alignments that cover $<70 \%$ of the read or fail to satisfy the gene-specific species-level percent identity cut-offs were discarded.

\section{Brain gene expression analysis}

Total RNA was extracted from individual brains using the Quick-RNA MiniPrep kit (Zymo; Irvine, CA, USA). RNA degradation and contamination were monitored on $1 \%$ agarose gels, and the purity was checked with the NanoPhotometer spectrophotometer (IMPLEN; CA, generated using NEBNext Ultra RNA Library Prep Kit for Illumina (New England BioLabs; Ipswich, MA, USA) and index codes were added to attribute sequences to each sample. The clustering of the index-coded samples was performed on a cBot Cluster Generation System using TruSeq PE Cluster Kit v3-cBot-HS (Illumina; San Diego, CA, USA), and the library preparations were then sequenced on an Illumina NovaSeq 6000 platform (Illumina; San Diego, CA, USA) and 150 bp paired-end reads were generated. Sequencing quality of individual samples was assessed using FastQC v0.11.5 with default parameters. An index of 
the bee reference genome (Amel_HAv3.1) was built using HISAT2 v2.0.5 ${ }^{86}$, and the FastQC

671 trimmed reads were then aligned to the built index using HISAT2 v2.1.0 with default

672 parameters. Gene expression was quantified using HTSeq v0.7.2 ${ }^{87}$ with mode 'union', only reads mapping unambiguously to a single gene are counted, whereas reads aligned to multiple positions or overlapping with more than one gene are discarded. If it were counted for both genes, the extra reads from the differentially expressed gene may cause the other gene to be wrongly called differentially expressed, so we chose 'union' mode. We modeled read counts following a negative binomial distribution with normalized counts and dispersion. The proportion of the gene counts in the sample to the concentration of cDNA was scaled by a normalization factor using the median-of-ratios method. The variability between replicates is modeled by the dispersion parameter using empirical Bayes shrinkage estimation. For each gene, we fit a generalized linear model to get the overall expression strength of the gene and the log 2-fold change between $\mathrm{CV}, \mathrm{MC}$, and MF groups. For significance testing, differential gene expression is determined by the Wald test. The resulting p-values were corrected for multiple comparisons using the Benjamini-Hochberg FDR method ${ }^{89}$. Genes with an adjusted P-value $<0.05$ and $\mid \log _{2}$ FoldChange $\mid>1$ were assigned as differentially expressed.

To get a better annotation of the honeybee reference genome, we re-annotate it using eggNOG-mapper v5.090. 6,269 out of 12,375 honeybee genes were successfully assigned to a KO entry with the 'diamond' mode, and the hierarchy information of the KEGG metabolic

691 pathway was extracted. Functional analysis of differentially expressed genes was performed 692 based on KEGG Orthologue (KO) markers. The percentages of KO markers belong to each 693 category (KEGG Class at level 3) out of total MC-, CV-, and MF-enriched KO markers were 
designated as a comparison parameter. The significance level was calculated by Fisher's exact test using clusterProfiler v3.10.191.

$$
\varphi=\frac{\frac{I}{l_{I}}}{\frac{I}{l_{I}}+\frac{S}{l_{S}}}
$$

, where S and I are the numbers of reads mapped to the junction supporting skipping and inclusion form, respectively. Effective length 1 is used for normalization. The PSI value was calculated for several classes of alternative splicing events, including skipped exon (SE), alternative 5' splice site (A5SS), alternative 3' splice site (A3SS), mutually exclusive exons (MXE), and retained introns (RI). Events with $\mathrm{p}<0.05$ were considered differentially spliced across gnotobiotic bees and microbiota-free bees.

To find the overlaps between the differentially expressed or spliced genes of bee brain and those from humans autism spectrum disorders, a total of 3,531 high-quality reference protein sequences corresponding to 948 known autism risk genes (SFARI: https://gene.sfari.org/, SPARK for Autism: http://sparksf.s3.amazonaws.com/SPARK_gene_list.pdf) were aligned against the protein sequences of

712 honeybee genome using BLASTP ${ }^{93}$ with two-way best matching strategy. In total, 649

713 autism protein sequences obtained a match (Similarity $>30 \%$ and e-value $<0.000394$ ). Then

714 we calculated the intersection of the autism risk genes and the differentially expressed or

715 spliced genes between bacteria colonized bees and MF bees $(p<0.05)$. 


\section{Brain proteome analysis.}

717 The proteome analysis was performed as described by Meng et al. ${ }^{94}$. Briefly, three replicates

718 per treatment group were analyzed for each group of bees. 20 dissected honeybee brains were pestle ground, sonicated, and cooled on ice for $30 \mathrm{~min}$ in a lysis buffer $(8 \mathrm{M}$ urea, $2 \mathrm{M}$ thiourea, 4\% 3-((3-cholamidopropyl) dimethylammonio)-1-propanesulfonate acid (CHAPS), $20 \mathrm{mM}$ tris-base, $30 \mathrm{mM}$ dithiothreitol (DDT)). The homogenate was centrifuged at 12,000 $\mathrm{g}$ and $4{ }^{\circ} \mathrm{C}$ for $15 \mathrm{~min}$, followed by supernatant recovery. Then 4 volumes of ice-cold acetone were added for $30 \mathrm{~min}$ to precipitate protein. The protein pellets were collected after centrifugation $\left(8,000 \mathrm{~g}, 4^{\circ} \mathrm{C}\right.$ for $\left.15 \mathrm{~min}\right)$, then dried at room temperature, and dissolved in 40 $\mathrm{mM} \mathrm{NH}_{4} \mathrm{HCO}_{3}$. To prevent reformation of disulfide bonds, the dissolved protein samples were incubated with $100 \mathrm{mM}$ of DDT (DDT/protein $(\mathrm{V}: \mathrm{V}=1: 10))$ for $1 \mathrm{~h}$ and then alkylated with $50 \mathrm{mM}$ of iodoacetamide (IAA) (DDT/IAA (V: V=1:5)) for $1 \mathrm{~h}$ in the dark. Finally, the resultant protein was digested with trypsin (enzyme: protein (W: $\mathrm{W}=1: 50)$ ) at $37^{\circ} \mathrm{C}$ for $14 \mathrm{~h}$. After digestion, the enzymatic reaction was stopped by adding $1 \mu \mathrm{L}$ of formic acid into the mixture. The digested peptides were centrifuged at $13,000 \mathrm{~g}$ and $4^{\circ} \mathrm{C}$ for $10 \mathrm{~min}$. The supernatant was recovered and extracted using a SpeedVac system (RVC 2-18, Marin Christ; Osterod, Germany) for subsequent LC-MS/MS analysis. Scientific, Waltham, MA, USA) on a Q Exactive HF mass spectrometer (Thermo Fisher Scientific). Peptides were separated on an analytical column packed with $2 \mu \mathrm{m}$ Aqua C18 beads ( $15 \mathrm{~cm}$ long, $50 \mu \mathrm{m}$ inner diameter, Thermo Fisher Scientific) at a flow rate of 350 $\mathrm{nL} / \mathrm{min}$, using a 120 -min gradient $(2 \%$ (vol $/ \mathrm{vol})$ to $10 \%$ (vol $/ \mathrm{vol})$ acetonitrile with $0.1 \%$ (vol/vol) formic acid). The Q Exactive was operated in the data-dependent mode with the following settings: 70000 resolution, $350-1,600 \mathrm{~m} / \mathrm{z}$ full scan, Top 20, and a $2 \mathrm{~m} / \mathrm{z}$ isolation window. Identification and label-free quantification of peptides were done with PEAKS 
Studio X+ (Bioinformatics Solutions Inc.; Waterloo, ON, Canada) against the sequence database (21,780 protein sequences of Apis mellifera), coupled with a common repository of adventitious proteins database (cRAP, https://www.thegpm.org/dsotw_2012.html). The search parameters were: parent ion tolerance, $15 \mathrm{ppm}$; fragment tolerance, $0.05 \mathrm{Da}$; enzyme, trypsin; maximum missed cleavages, 3 ; fixed modification, carbamidomethyl $(\mathrm{C},+57.02$ Da); and variable modification, oxidation $(\mathrm{M},+15.99 \mathrm{Da})$. A protein was confidently identified only if it contained at least one unique peptide with at least two spectra, applying a threshold of false discovery rate (FDR) $\leq 1.0 \%$ by a fusion-decoy database searching strategy (PMID: 22186715). Proteins significantly differential between groups were identified using ANOVA ( $p$-value $<0.05$ and a fold change of $\geq 1.5$ ).

The functional gene ontology (GO) term and pathway were assessed using ClueGOv2.5.5, Cytoscape plug-in software (http://www.ici.upmc.fr/cluego/). The analysis was performed by comparing an input data set of identified proteins to all functionally annotated GO categories in the entire genome of Apis mellifera from UniProt. The significantly enriched GO terms in cellular component (CC), molecular function (MF), biological processes (BPs) and pathways were reported using a two-sided hyper-geometric test and only a $p$-value $\leq 0.05$ was considered. Then, Bonferroni step-down was used to correct the p-value to control FDR. Functional grouping of the terms was based on the GO hierarchy. The tree level was ranged from 3 to 8 , and the kappa score level was 0.4 .

\section{Targeted metabolomics for brain neurotransmitters.}


$20 \mu \mathrm{L}$ ultrapure water were added and the tubes were vortexed thoroughly. Metabolites were sonicated in an ice-water bath for $30 \mathrm{~min}$, followed by subsiding at $-20{ }^{\circ} \mathrm{C}$ for $2 \mathrm{~h}$. addition of $10 \mu \mathrm{L}$ sodium carbonate solution $(100 \mathrm{mM})$ and $10 \mu \mathrm{L} 2 \%$ benzoyl chloride acetonitrile. Then $1.6 \mu \mathrm{L}$ internal standard and $20 \mu \mathrm{L} 0.1 \%$ formic acid were added, and the samples were centrifuged $\left(14,000 \mathrm{~g} \times 5 \mathrm{~min}\right.$ at $\left.4{ }^{\circ} \mathrm{C}\right) .40 \mu \mathrm{L}$ of the supernatants were transferred to an auto-sampler vial for downstream UHPLC-MS/MS analysis. Serotonin hydrochloride, $\gamma$-aminobutyric acid, dopamine hydrochloride, tyramine, and octopamine hydrochloride (Aladdin; Shanghai, China) derivatized with benzoyl chloride (SigmaAldrich; Saint Louis, MO, USA) were used for the construction of the calibration standard curve. The internal standards mixture ( $\gamma$-aminobutyric acid, dopamine hydrochloride, serotonin hydrochloride, tyramine, and octopamine hydrochloride derivatized with benzoyl chloride-d5 (Sigma-Aldrich; Saint Louis, MO, USA) ${ }^{95}$ of the corresponding concentration were prepared, respectively. USA), and the samples were analyzed on the QTRAP 6500 LC-MS/MS system (AB Sciex; temperature was set at $40{ }^{\circ} \mathrm{C}$, and the auto-sampler temperature was set at $4{ }^{\circ} \mathrm{C}$.

785 Chromatographic separation was achieved using a $0.30 \mathrm{ml} / \mathrm{min}$ flow rate and a linear gradient of 0 to $2 \%$ B within 2 min; $2 \%-98 \%$ B in 9 min, followed by $98 \%$ B for 2 min and equilibration for $2 \mathrm{~min}$. Solvent A is $0.1 \%$ formic acid and solvent B is acetonitrile. For all multiple reaction monitoring (MRM) experiments, 6500 QTrap acquisition parameters were 
as follows: $5000 \mathrm{~V}$ Ion-spray voltage, curtain gas setting of 35 and nebulizer gas setting of 60, temperature at $400^{\circ} \mathrm{C}$. Raw data were analyzed using Skyline ${ }^{96}$.

\section{Quasi-Targeted metabolomics analysis.}

792

793

794

795

796

797

798

799

800

801

802

803

804

805

806

807

808

809

810

811

812

Hemolymph and gut homogenate metabolites were determined by quasi-targeted metabolomics by HPLC-MS/MS. Gut samples (100mg) were individually grounded with liquid nitrogen and the homogenate was resuspended with prechilled $500 \mu \mathrm{L} 80 \%$ methanol and $0.1 \%$ formic acid by well vortexing. $50 \mu \mathrm{L}$ of hemolymph samples were mixed with 400 $\mu \mathrm{L}$ prechilled methanol by vortexing. All samples were incubated on ice for $5 \mathrm{~min}$ and then centrifuged at $15,000 \times \mathrm{g}$, at $4^{\circ} \mathrm{C}$ for $10 \mathrm{~min}$. The supernatant was diluted to a final concentration containing 53\% methanol by LC-MS grade water. The samples were then transferred to a fresh vial and centrifuged at $15,000 \times \mathrm{g}, 4^{\circ} \mathrm{C}$ for $20 \mathrm{~min}$. Finally, the supernatant was injected into the LC-MS/MS system, and the analyses were performed using an ExionLC AD system (SCIEX) coupled with a QTRAP 6500+ mass spectrometer (SCIEX). Samples were injected onto a BEH C8 Column $(100 \mathrm{~mm} \times 2.1 \mathrm{~mm} \times 1.9 \mu \mathrm{m})$ using a 30-min linear gradient at a flow rate of $0.35 \mathrm{~mL} / \mathrm{min}$ for the positive polarity mode. Eluent A was $0.1 \%$ formic acid-water and eluent B is $0.1 \%$ formic acid-acetonitrile. The solvent gradient was set as follows: 5\% B, $1 \mathrm{~min} ; 5-100 \% \mathrm{~B}, 24.0 \mathrm{~min} ; 100 \% \mathrm{~B}, 28.0$ $\min ; 100-5 \%$ B, $28.1 \mathrm{~min} ; 5 \%$ B, 30 min. QTRAP 6500+ mass spectrometer was operated in positive polarity mode with curtain gas of 35 psi, collision gas of Medium, ion spray voltage of $5500 \mathrm{~V}$, temperature of $500^{\circ} \mathrm{C}$, ion source gas of $1: 55$, and ion source gas of 2:55. For negative ion mode, samples were injected onto aHSS T3 Column $(100 \mathrm{~mm} \times 2.1 \mathrm{~mm})$ using a $25-\mathrm{min}$ linear gradient at a flow rate of $0.35 \mathrm{~mL} / \mathrm{min}$. The solvent gradient was set as follows: $2 \% \mathrm{~B}, 1 \mathrm{~min} ; 2 \%-100 \% \mathrm{~B}, 18.0 \mathrm{~min} ; 100 \% \mathrm{~B}, 22.0 \mathrm{~min} ; 100 \%-5 \% \mathrm{~B}, 22.1 \mathrm{~min}$; 5\% B, 25 min. QTRAP 6500+ mass spectrometer was operated in negative polarity mode 
813 with curtain gas of $35 \mathrm{psi}$, collision gas of medium, ion spray voltage of $-4500 \mathrm{~V}$,

814 temperature of $500^{\circ} \mathrm{C}$, ion source gas of 1:55, and ion source gas of 2:55.

Detection of the experimental samples using MRM was based on Novogene in-house

816 database. Q3 (daughter) was used for the quantification. Q1 (parent ion), Q3, retention time,

817 declustering potential, and collision energy were used for metabolite identification. Data

818 files generated by HPLC-MS/MS were processed with SCIEX OS (version 1.4) to integrate

819 and correct the peaks. A total of 326 compounds were identified in the hemolymph samples.

820 Metabolomics data analysis was then performed using MetaboAnalyst 4.0 ${ }^{97}$.

821 Weighted gene co-expression network analysis (WGCNA)

R software package WGCNA $1.69^{98}$ was used to identify key phenotype-related metabolic modules based on correlation patterns. The Pearson correlation matrix was

824 calculated for all possible metabolite pairs and then transformed into an adjacency matrix

825 with a soft thresholding power setting to 5 for the best topological overlap matrix. A

826 dynamic tree cut algorithm was used to detect groups of highly correlated metabolites. The

827 minimum module size was set to 14 and the threshold for merging module was set to 0.25 as

828 default. Each module was assigned a unique color and contained a unique set of metabolites.

829 After obtaining modules from each group, module eigenmetabolite was calculated with the

830 "ModuleEigengenes" function. Association analysis between a module and the trait of each

831 group was performed using the function of "corPvalueStudent" based on the module

832 eigenmetabolite. $\mathrm{p}<0.01$ was set for statistical significance. Metabolites in each module

833 were annotated on the KEGG Database and classified into major categories using

834 MetaboAnalyst $4.0^{97}$ for enrichment analysis. Finally, the network connections among

835 metabolites in modules were visualized using Cytoscape 3.7.099. 


\section{Statistical analysis}

Comparison of the learning and memory results was tested by Chi-squared test using

838 GraphPad Prism 8.2.0 software. Comparisons of the distribution of gustatory response score,

839 neurotransmitters, normalized and raw metabolite data of different bacterial colonized

840 groups were made by Mann-Whitney $u$ test using GraphPad Prism 8.2.0 software. The exact

841 value of $\mathrm{n}$ representing the number of groups in the experiments described was indicated in

842 the figure legends. Any additional technical replicates are described within the Methods and

843 the Results.

\section{Data Availability}

845 The raw data for outdoor honeybee gut microbiome shotgun sequencing has been

846 deposited under BioProject PRJNA670603. The accession numbers for the RNA sequencing

847 data are PRJNA670620 and PRJNA668910. The proteomic data has been deposited to the

848 Proteome Xchange Consortium with the dataset identifier PXD022304.

\section{Code availability}

851 on GitHub at: https://github.com/ZijingZhang93/bee BGA.git. 
A

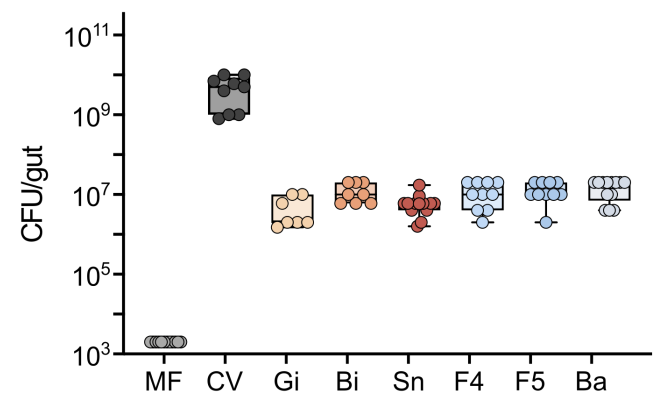

$\mathrm{B}$
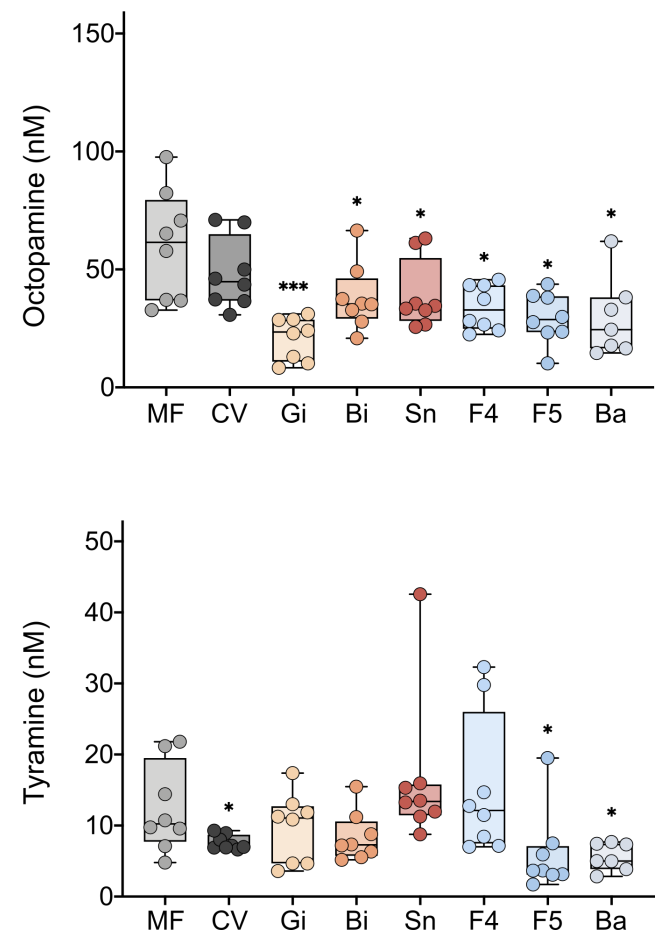

854 Supplementary Fig. 1. Gut microbiota impacts the concentrations of tyramine and

855 octopamine in the honeybee brain. (a) Boxplots of the total CFU per gut estimated by

856 bacteria culture for MF and mono-colonized bees, or by qPCR for the CV group. (b-c)

857 Concentrations of $(\mathbf{b})$ tyramine and (c) octopamine in MF $(n=8), \mathrm{CV}(\mathrm{n}=8)$, and mono-

858 colonized ( $\mathrm{n}=8$, except $\mathrm{n}=7$ for Ba group) bee brains. Differences between bacteria-

859 colonized bees and the MF group were tested by Mann-Whitney $u$ test $\left({ }^{*} \mathrm{p}<0.1,{ }^{* *} \mathrm{p}<\right.$

$860 \quad 0.01, * * * p<0.001)$. Error bars represent min and max. 
A

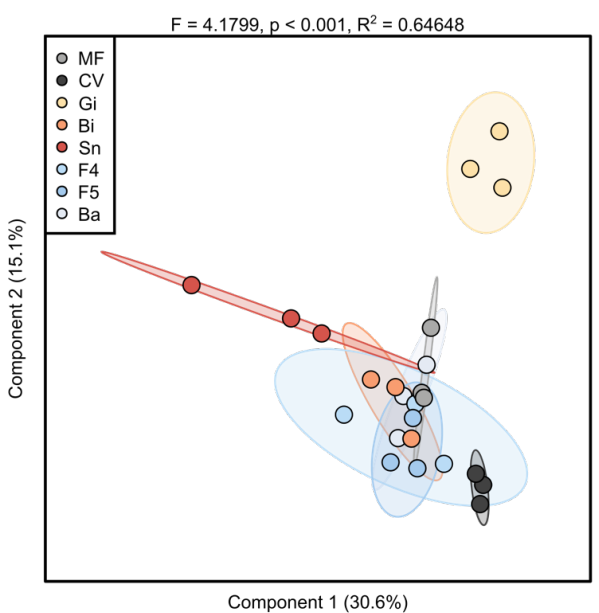

B
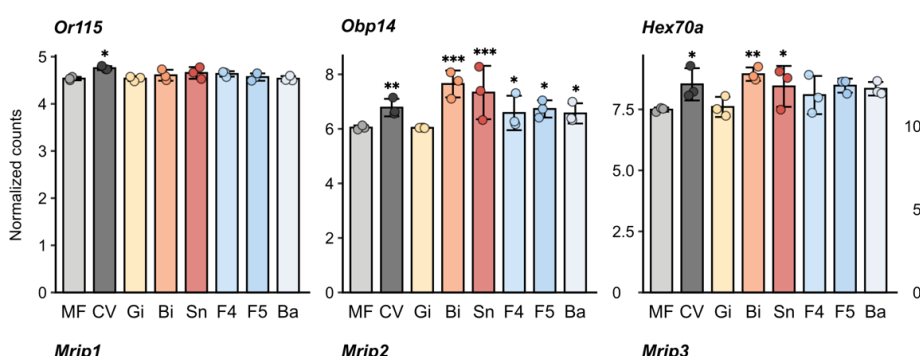

$v g$
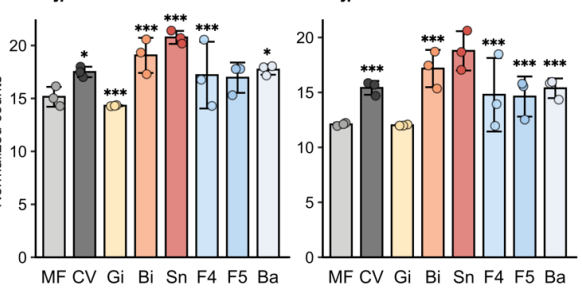

Mrjp4

Mrjp

Mrjp 7
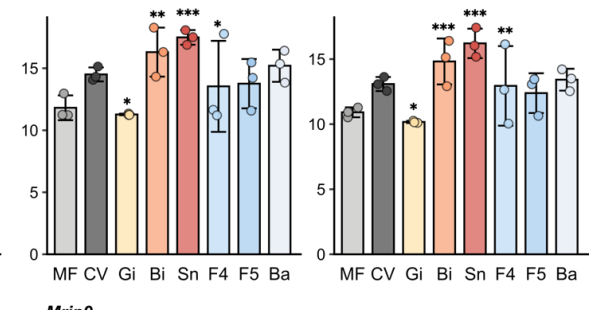

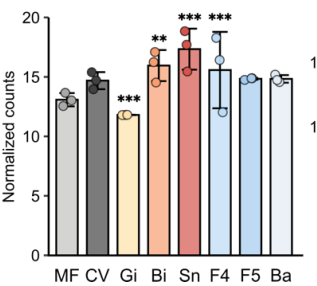
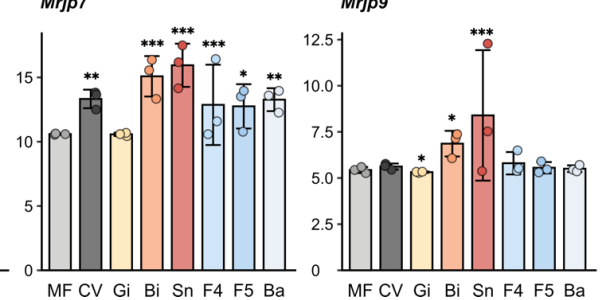

\section{Supplementary Fig. 2. Gut microbiome impacts gene expression in the honeybee}

863 brain. (a) Sparse PLS-DA based on normalized gene expression in the brain of microbiota-

864 free and bacteria-colonized bees. Group differences were tested by PERMANOVA. (b)

865 Relative expression levels of differentially expressed genes in the brains of different bee

866 groups. Differences between bacteria-colonized bees and the MF group were tested by

867 Wald test with Benjamini-Hochberg correction $(* \mathrm{FDR}<0.05, * * \mathrm{FDR}<0.01, * * * \mathrm{FDR}<$

868 0.001). Data are shown as mean \pm SEM. 
A

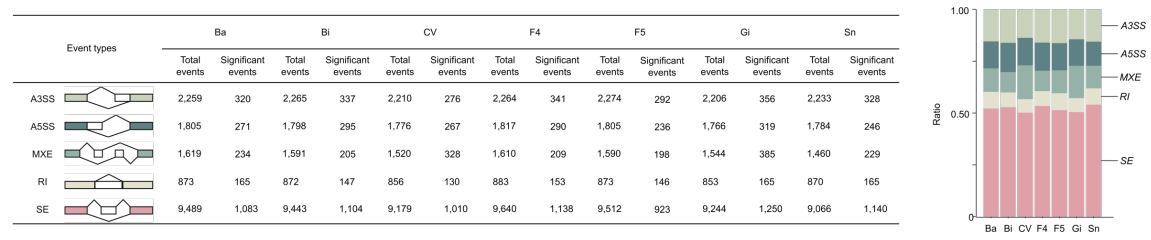

B
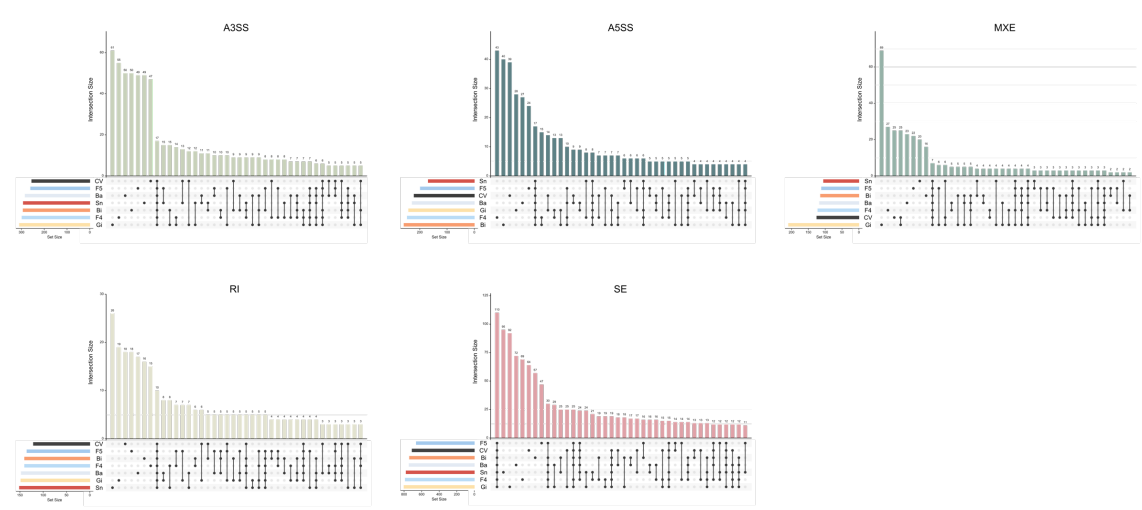

C

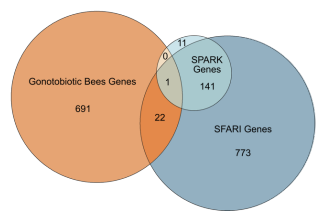

870 Supplementary Fig. 3. Gut microbiome impacts spliced genes in the honeybee brain.

871 (a) Numbers of the differential alternative splicing events in the brains of bacteria-

872 colonized bees compared to MF bees. Stacked column graph shows the relative abundance

873 of different types of alternative splicing events in each group. A3SS, alternative 3' splice

874 site; A5SS, alternative 5' splice site; MXE, mutually exclusive exon; RI, retained introns;

875 SE, skipped exon. (b) UpSet plots showing the intersections of alternative splicing (AS)

876 events associated with different bacteria-colonized groups. The dots and lines on the

877 bottom right represent which intersection is shown by the bar plots above. The size of

878 intersections is given above the bar plot. The total amount of different types of events for

879 each bee group is given to the left of the intersection diagram. (c) Venn diagram of

880 differentially expressed genes in the brains between MF and CV/mono-colonized bees

881 (FDR $<0.05)$, and their overlap with the SPARK and SFARI Gene datasets. Differentially expressed genes were identified by Wald test with Benjamini-Hochberg correction. 
A

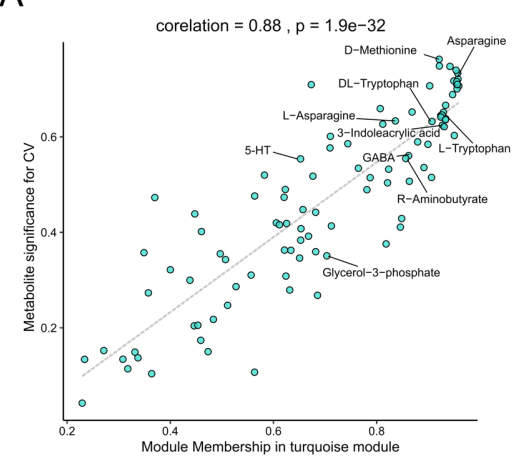

C

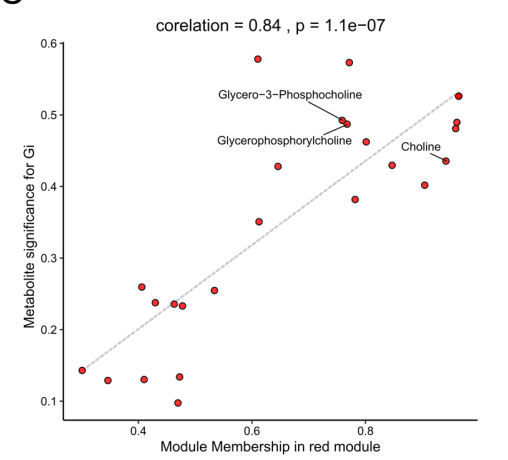

E
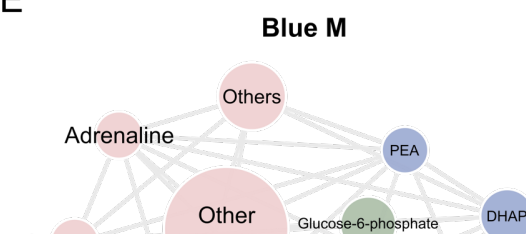

Glutamine

883
B

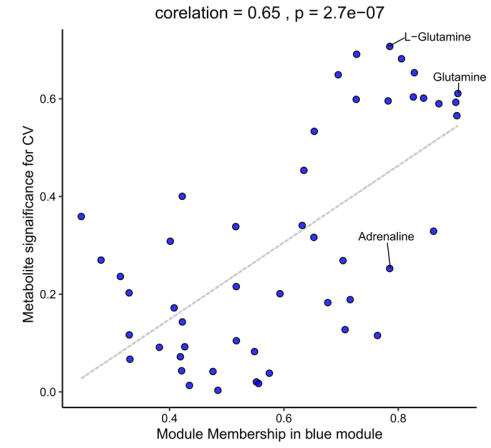

D

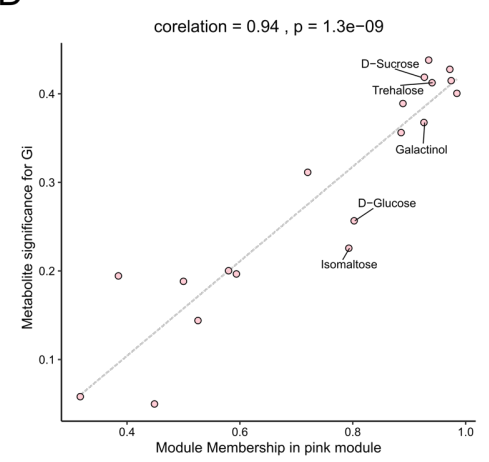

\section{Supplementary Fig. 4. Identification of intramodular connectivity and network}

885 analysis. (a-d) The correlation analysis between metabolite-module connectivity ( $\mathrm{X}$ axis)

886 and metabolites significantly correlated with different bee groups ( $\mathrm{Y}$ axis): (a) turquoise $\mathrm{M}$

887 and (b) blue $\mathrm{M}$ with $\mathrm{CV}$ group; (c) red $\mathrm{M}$ and (d) pink M with Gi group. (e) Network

888 diagrams of differential metabolites in the blue and pink M. Circle colors indicate different

889 classes of metabolites in each module, and the circle size is proportional to the total

890 abundance of metabolites in each module. 
A
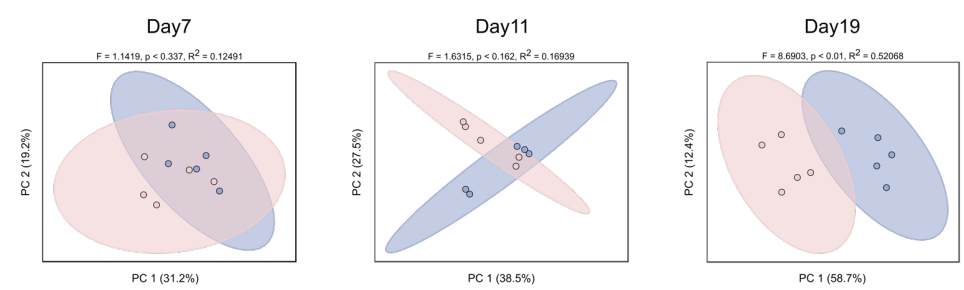

OControl

B
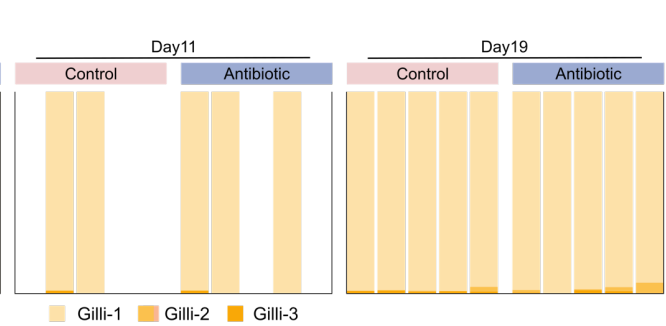

C

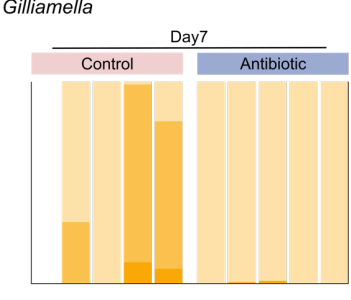

Gilli-1 Gilli-2 ㅁilli-3

Bifidobacterium

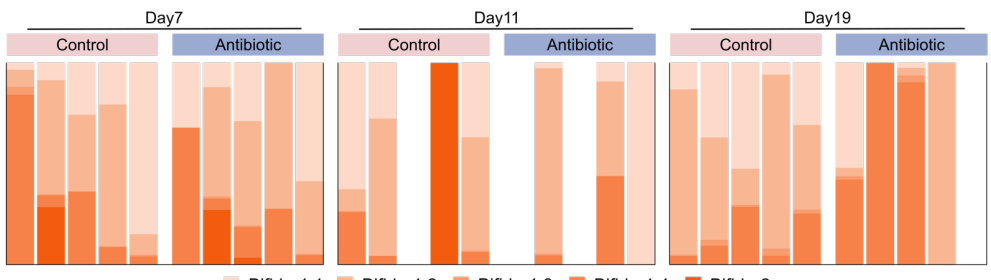

D

Lactobacillus Firm4
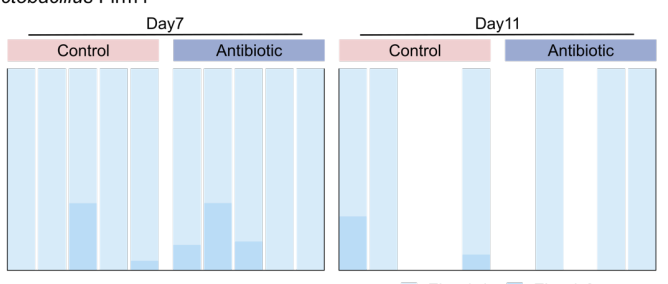

$E$

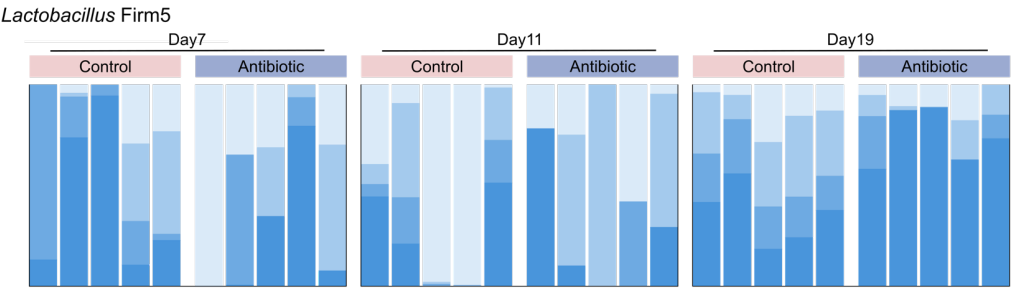

892 Supplementary Fig. 5. Antibiotic treatment affects the gut microbiota of honeybees.

893 (a) Principal coordinate analysis of Bray-Curtis dissimilarity of gut community

894 compositions of control and antibiotic-treated bees. Group differences were tested by

895 PERMANOVA. (b-e) Compositions at SDP-level for four core bee gut members: (b) 
897 Supplementary Data 1. Normalized gene expression levels in brains of microbiota-free

898 and bacteria-colonized bees.

899 Supplementary Data 2. Alternative splicing events in brains of microbiota-free and

900 bacteria-colonized bees.

901 Supplementary Data 3. Identification and biological function analysis of proteins

902 expressed in brains of microbiota-free and conventional bees.

903 Supplementary Data 4. Raw data of all metabolites abundance in the hemolymph of

904 microbiota-free and bacteria-colonized bees, and in the colon of antibiotic-treated and

905 control bees.

906 Supplementary Data 5. Hemolymph metabolomic WGCNA module analysis of

907 microbiota-free and bacteria-colonized bees.

908 Supplementary Data 6. The list of genomes of bacterial isolates in the database for

909 MIDAS profiling.

910 Supplementary Movie 1. Olfactory learning and memory test. 


\section{References}

$9121 \quad$ Cryan, J. F. \& Dinan, T. G. Mind-altering microorganisms: the impact of the gut

913 microbiota on brain and behaviour. Nat. Rev. Neurosci. 13, 701-712, (2012).

9142 Hsiao, E. Y. et al. Microbiota modulate behavioral and physiological abnormalities

915 associated with neurodevelopmental disorders. Cell 155, 1451-1463, (2013).

9163 Taj, A. \& Jamil, N. Bioconversion of tyrosine and tryptophan derived biogenic amines

917 by neuropathogenic bacteria. Biomolecules $\mathbf{8}, 10,(2018)$.

9184 Marques, T. M. et al. Influence of GABA and GABA-producing Lactobacillus brevis

919 DPC 6108 on the development of diabetes in a streptozotocin rat model. Benef. Microbes 7 ,

920 409-420, (2016).

9215 Clarke, G. et al. The microbiome-gut-brain axis during early life regulates the

922 hippocampal serotonergic system in a sex-dependent manner. Mol. Psychiatry 18, 666-673, 923 (2013).

9246 Nankova, B. B., Agarwal, R., MacFabe, D. F. \& La Gamma, E. F. Enteric bacterial 925 metabolites propionic and butyric acid modulate gene expression, including CREB-dependent 926 catecholaminergic neurotransmission, in PC12 cells--possible relevance to autism spectrum 927 disorders. PLoS One 9, e103740, (2014).

9287 Sharon, G. et al. Human gut microbiota from autism spectrum disorder promote 929 behavioral symptoms in mice. Cell 177, 1600-1618 e1617, (2019).

9308 Bobay, L. M. \& Raymann, K. Population genetics of host-associated microbiomes. 931 Curr. Mol. Bio. Rep. 5, 128-139, (2019).

9329 Scheiner, R. et al. Standard methods for behavioural studies of Apis mellifera. $J$. 933 Apicult. Res. 52, 1-58, (2013).

$93410 \quad$ Kwong, W. K. \& Moran, N. A. Gut microbial communities of social bees. Nat. Rev. 935 Microbiol. 14, 374-384, (2016).

$936 \quad 11 \quad$ Bonilla-Rosso, G. \& Engel, P. Functional roles and metabolic niches in the honey bee 937 gut microbiota. Curr. Opin. Microbiol. 43, 69-76, (2018).

$93812 \quad$ Ellegaard, K. M. \& Engel, P. Genomic diversity landscape of the honey bee gut 939 microbiota. Nat. Commun. 10, 446, (2019).

94013 Kešnerová, L. et al. Disentangling metabolic functions of bacteria in the honey bee 941 gut. PLoS Biol. 15, e2003467, (2017).

94214 Zheng, H., Powell, J. E., Steele, M. I., Dietrich, C. \& Moran, N. A. Honeybee gut 943 microbiota promotes host weight gain via bacterial metabolism and hormonal signaling. Proc. 944 Natl. Acad. Sci. U S A 114, 4775-4780, (2017).

94515 Zheng, H., Steele, M. I., Leonard, S. P., Motta, E. V. S. \& Moran, N. A. Honey bees as 946 models for gut microbiota research. Lab Anim. (NY) 47, 317-325, (2018). 
$94716 \quad$ Zheng, H. et al. Division of labor in honey bee gut microbiota for plant polysaccharide

948 digestion. Proc. Natl. Acad. Sci. US A 116, 25909-25916, (2019).

94917 Harris, J. W. \& Woodring, J. Effects of stress, age, season, and source colony on

950 levels of octopamine, dopamine and serotonin in the honey bee (Apis mellifera L.) brain. $J$.

951 Insect Physiol. 38, 29-35, (1992).

95218 Farina, W. M., Grüter, C. \& Arenas, A. in Honeybee Neurobiology and Behavior

953 (eds C. Galizia, D. Eisenhardt, \& M. Giurfa) 89-101 (Springer, 2012).

95419 Laloi, D., Gallois, M., Roger, B. \& Pham-Delegue, M. H. Changes with age in

955 olfactory conditioning performance of worker honey bees (Apis mellifera). Apidologie 32,

956 231-242,(2001).

95720 Simcock, N. K., Gray, H., Bouchebti, S. \& Wright, G. A. Appetitive olfactory learning 958 and memory in the honeybee depend on sugar reward identity. J. Insect Physiol. 106, 71-77, 959 (2018).

96021 Giurfa, M. Behavioral and neural analysis of associative learning in the honeybee: a

961 taste from the magic well. J. Comp. Physiol. A. 193, 801-824, (2007).

96222 Mercer, A. R. \& Menzel, R. The effects of biogenic amines on conditioned and 963 unconditioned responses to olfactory stimuli in the honeybee Apis mellifera. J. Comp.

964 Physiol. 145, 363-368, (1982).

96523 Scheiner, R., Plückhahn, S., Oney, B., Blenau, W. \& Erber, J. Behavioural

966 pharmacology of octopamine, tyramine and dopamine in honey bees. Behav. Brain Res. 136,

967 545-553, (2002).

96824 Stopfer, M., Bhagavan, S., Smith, B. H. \& Laurent, G. Impaired odour discrimination

969 on desynchronization of odour-encoding neural assemblies. Nature 390, 70-74, (1997).

97025 El Hassani, A. K., Dacher, M., Gauthier, M. \& Armengaud, C. Effects of sublethal

971 doses of fipronil on the behavior of the honeybee (Apis mellifera). Pharmacol. Biochem.

972 Behav. 82, 30-39, (2005).

97326 El Hassani, A. K., Dupuis, J. P., Gauthier, M. \& Armengaud, C. Glutamatergic and

974 GABAergic effects of fipronil on olfactory learning and memory in the honeybee. Invert.

975 Neurosci. 9, 91-100, (2009).

97627 Li, Z. G. et al. Brain transcriptome of honey bees (Apis mellifera) exhibiting impaired

977 olfactory learning induced by a sublethal dose of imidacloprid. Pestic. Biochem. Physiol. 156,

978 36-43, (2019).

97928 Vogt, R. G., Prestwich, G. D. \& Lerner, M. R. Odorant-binding-protein subfamilies 980 associate with distinct classes of olfactory receptor neurons in insects. J. Neurobiol. 22, 74$98184,(1991)$.

$98229 \quad$ Ullah, R. M. K. et al. An odorant binding protein (SaveOBP9) involved in 983 chemoreception of the wheat aphid Sitobion avenae. Int. J. Mol. Sci. 21, 8331, (2020). 
984

985

986

987

988

989

990

991

992

993

994

995

996

997

998

999

1000

1001

1002

1003

1004

1005

1006

1007

1008

1009

1010

1011

1012

1013

1014

1015

1016

1017

1018

1019

1020

30 Drapeau, M. D., Albert, S., Kucharski, R., Prusko, C. \& Maleszka, R. Evolution of the Yellow/Major Royal Jelly Protein family and the emergence of social behavior in honey bees. Genome Res. 16, 1385-1394, (2006).

31 Nunes, F. M. F., Ihle, K. E., Mutti, N. S., Simoes, Z. L. P. \& Amdam, G. V. The gene vitellogenin affects microRNA regulation in honey bee (Apis mellifera) fat body and brain. $J$. Exp. Biol. 216, 3724-3732, (2013).

32 Martins, J. R., Nunes, F. M. F., Cristino, A. S., Simoes, Z. P. \& Bitondi, M. M. G. The four hexamerin genes in the honey bee: structure, molecular evolution and function deduced from expression patterns in queens, workers and drones. BMC Mol. Biol. 11, 23, (2010).

33 Mergenthaler, P., Lindauer, U., Dienel, G. A. \& Meisel, A. Sugar for the brain: the role of glucose in physiological and pathological brain function. Trends Neurosci. 36, 587597, (2013).

34 Shpigler, H. Y. et al. Deep evolutionary conservation of autism-related genes. Proc. Natl. Acad. Sci. U S A 114, 9653-9658, (2017).

35 Voineagu, I. et al. Transcriptomic analysis of autistic brain reveals convergent molecular pathology. Nature 474, 380-384, (2011).

36 Abrahams, B. S. et al. SFARI Gene 2.0: a community-driven knowledgebase for the autism spectrum disorders (ASDs). Mol. Autism 4, 36, (2013).

37 Kordeli, E. \& Bennett, V. Distinct ankyrin isoforms at neuron cell-bodies and nodes of Ranvier resolved using erythrocyte ankyrin deficient mice. J. Cell Biol. 114, 1243-1259, (1991).

38 Willsey, A. J. et al. Coexpression networks implicate human midfetal deep cortical projection neurons in the pathogenesis of autism. Cell 155, 997-1007, (2013).

39 Kaeser, P. S., Deng, L. B., Fan, M. M. \& Sudhof, T. C. RIM genes differentially contribute to organizing presynaptic release sites. Proc. Natl. Acad. Sci. U S A 109, 1183011835, (2012).

40 Sialana, F. J. et al. Proteome changes paralleling the olfactory conditioning in the forager honey bee and provision of a brain proteomics dataset. Proteomics 19, e1900094, (2019).

41 Gauthier, M. \& Grünewald, B. in Honeybee Neurobiology and Behavior (eds C. Galizia, D. Eisenhardt, \& M. Giurfa) 155-169 (Springer, 2012).

42 Grünewald, B. in Honeybee Neurobiology and Behavior (eds C. Galizia, D. Eisenhardt, \& M. Giurfa) 185-198 (Springer, 2012).

43 Engel, P., Martinson, V. G. \& Moran, N. A. Functional diversity within the simple gut microbiota of the honey bee. Proc. Natl. Acad. Sci. US A 109, 11002-11007, (2012).

44 Agus, A., Planchais, J. \& Sokol, H. Gut microbiota regulation of tryptophan metabolism in health and disease. Cell Host Microbe 23, 716-724, (2018). 
$102145 \quad$ Wlodarska, M. et al. Indoleacrylic acid produced by commensal Peptostreptococcus species suppresses inflammation. Cell Host Microbe 22, 25-37.e26, (2017).

102346 Zheng, P. et al. The gut microbiome modulates gut-brain axis glycerophospholipid 1024 metabolism in a region-specific manner in a nonhuman primate model of depression. Mol. 1025 Psychiatry. https://doi.org/10.1038/s41380-020-0744-2, (2020).

102647 Paerhati, Y. et al. Expression of $A m G R 10$ of the gustatory receptor family in honey 1027 bee is correlated with nursing behavior. PLoS One 10, e0142917, (2015).

102848 Liu, F. et al. lncRNA profile of Apis mellifera and its possible role in behavioural 1029 transition from nurses to foragers. Bmc Genomics 20, 393, (2019).

103049 Callery, P. S. \& Geelhaar, L. A. 1 - Piperideine as an in vivo precursor of the $\gamma-$ 1031 aminobutyric acid homologue 5 - aminopentanoic acid. J. Neurochem. 45, 946-948, (1985).

$103250 \quad$ Srinivasan, M. V. Honey bees as a model for vision, perception, and cognition. Annu. 1033 Rev. Entomol. 55, 267-284, (2010).

$103451 \quad$ Johnson, B. R. Division of labor in honeybees: form, function, and proximate 1035 mechanisms. Behav. Ecol. Sociobiol. 64, 305-316, (2010).

$103652 \quad$ Kapheim, K. M. et al. Caste-specific differences in hindgut microbial communities of 1037 honey bees (Apis mellifera). PLoS One 10, e0123911, (2015).

103853 Kešnerová, L. et al. Gut microbiota structure differs between honeybees in winter and 1039 summer. ISME J. 14, 801-814, (2020).

104054 Leger, L. \& McFrederick, Q. S. The gut-brain-microbiome axis in bumble bees.

1041 Insects 11, 517, (2020).

104255 McNally, G. P. \& Westbrook, R. F. Predicting danger: the nature, consequences, and 1043 neural mechanisms of predictive fear learning. Learn. Mem. 13, 245-253, (2006).

104456 Vergoz, V., Roussel, E., Sandoz, J. C. \& Giurfa, M. Aversive learning in honeybees 1045 revealed by the olfactory conditioning of the sting extension reflex. PLoS One 2, e288, 1046 (2007).

104757 Laska, M., Galizia, C. G., Giurfa, M. \& Menzel, R. Olfactory discrimination ability 1048 and odor structure-activity relationships in honeybees. Chem. Senses 24, 429-438, (1999).

104958 Harbo, J. R. Effect of population size on brood production, worker survival and honey 1050 gain in colonies of honeybees. J. Apicult. Res. 25, 22-29, (1986).

105159 DeNieu, M., Mounts, K. \& Manier, M. Two gut microbes are necessary and sufficient 1052 for normal cognition in Drosophila melanogaster. bioRxiv, 593723, (2019).

105360 Raymann, K., Bobay, L. M. \& Moran, N. A. Antibiotics reduce genetic diversity of 1054 core species in the honeybee gut microbiome. Mol. Ecol. 27, 2057-2066, (2018).

105561 Raymann, K., Shaffer, Z. \& Moran, N. A. Antibiotic exposure perturbs the gut 1056 microbiota and elevates mortality in honeybees. PLoS Biol. 15, e2001861, (2017). 
105762 Amdam, G. V., Fennern, E. \& Havukainen, H. in Honeybee Neurobiology and

1058 Behavior Vol., Dordrecht (eds C. Galizia, D. Eisenhardt, \& M. Giurfa) 17-29 (Springer, 1059 2012).

$106063 \quad$ Huang, S. K. et al. Evaluation of cage designs and feeding regimes for honey bee 1061 (Hymenoptera: Apidae) laboratory experiments. J. Econ. Entomol. 107, 54-62, (2014).

106264 Li, J. H. et al. Pollen reverses decreased lifespan, altered nutritional metabolism and 1063 suppressed immunity in honey bees (Apis mellifera) treated with antibiotics. J. Exp. Biol. 222, 1064 jeb202077, (2019).

106565 Kocher, S. D. et al. The genetic basis of a social polymorphism in halictid bees. Nat. 1066 Commun. 9, 4338, (2018).

106766 Sherwin, E., Bordenstein, S. R., Quinn, J. L., Dinan, T. G. \& Cryan, J. F. Microbiota 1068 and the social brain. Science 366, eaar2016, (2019).

106967 Vernier, C. L. et al. The gut microbiome defines social group membership in honey 1070 bee colonies. Sci. Adv. 6, eabd3431, (2020).

107168 Teseo, S. et al. The scent of symbiosis: gut bacteria may affect social interactions in 1072 leaf-cutting ants. Anim. Behav. 150, 239-254, (2019).

107369 Matsuura, K. Nestmate recognition mediated by intestinal bacteria in a termite, 1074 Reticulitermes speratus. Oikos 92, 20-26, (2001).

$107570 \quad$ Kałużna-Czaplińska, J., Gątarek, P., Chirumbolo, S., Chartrand, M. S. \& Bjørklund, 1076 G. How important is tryptophan in human health? Crit. Rev. Food Sci. Nutr. 59, 72-88, 1077 (2019).

107871 Lim, C. K. et al. Altered kynurenine pathway metabolism in autism: implication for 1079 immune-induced glutamatergic activity. Autism Res. 9, 621-631, (2016).

108072 Chen, K. et al. Drosophila histone demethylase KDM5 regulates social behavior through immune control and gut microbiota maintenance. Cell Host Microbe 25, 537-552, (2019).

108373 Ismail, N., Robinson, G. E. \& Fahrbach, S. E. Stimulation of muscarinic receptors mimics experience-dependent plasticity in the honey bee brain. Proc. Natl. Acad. Sci. US A 103, 207-211, (2006).

$108674 \quad$ Smith, D. B. et al. Insecticide exposure during brood or early-adult development 1087 reduces brain growth and impairs adult learning in bumblebees. Proc. Biol. Sci. 287, 1088 20192442, (2020).

108975 Kohno, H., Suenami, S., Takeuchi, H., Sasaki, T. \& Kubo, T. Production of knockout 1090 mutants by CRISPR/Cas9 in the European honeybee, Apis mellifera L. Zoolog. Sci. 33, 505$1091512,(2016)$.

109276 Leonard, S. P. et al. Genetic engineering of bee gut microbiome bacteria with a toolkit 1093 for modular assembly of broad-host-range plasmids. ACS Synth. Biol. 7, 1279-1290, (2018). 
$109477 \quad$ Kwong, W. K. \& Moran, N. A. Cultivation and characterization of the gut symbionts of honey bees and bumble bees: description of Snodgrassella alvi gen. nov., sp. nov., a member of the family Neisseriaceae of the Betaproteobacteria, and Gilliamella apicola gen. nov., sp. nov., a member of Orbaceae fam. nov., Orbales ord. nov., a sister taxon to the order 'Enterobacteriales' of the Gammaproteobacteria. Int. J. Syst. Evol. Microbiol. 63, 2008-2018, 1099 (2013).

$110078 \quad$ Zufelato, M. S., Lourenco, A. P., Simoes, Z. L., Jorge, J. A. \& Bitondi, M. M. 1101 Phenoloxidase activity in Apis mellifera honey bee pupae, and ecdysteroid-dependent 1102 expression of the prophenoloxidase mRNA. Insect Biochem. Mol. Biol. 34, 1257-1268, 1103 (2004).

110479 Mota, T., Giurfa, M. \& Sandoz, J. C. Color modulates olfactory learning in honeybees 1105 by an occasion-setting mechanism. Learn. Mem. 18, 144-155, (2011).

$110680 \quad$ Guerrieri, F., Schubert, M., Sandoz, J. C. \& Giurfa, M. Perceptual and neural olfactory similarity in honeybees. PLoS Biol. 3, 718-732, (2005).

$110881 \quad$ Page, R. E., Jr., Erber, J. \& Fondrk, M. K. The effect of genotype on response thresholds to sucrose and foraging behavior of honey bees (Apis mellifera L.). J. Comp.

1110 Physiol. A. 182, 489-500, (1998).

111182 Mengoni Goñalons, C. \& Farina, W. M. Effects of sublethal doses of imidacloprid on 1112 young adult honeybee behaviour. PLoS One 10, e0140814, (2015).

111383 Huang, Z. Y. \& Robinson, G. E. Honeybee colony integration: worker-worker interactions mediate hormonally regulated plasticity in division of labor. Proc. Natl. Acad. Sci. U S A 89, 11726-11729, (1992).

111684 Jones, J. C. et al. The gut microbiome is associated with behavioural task in honey 1117 bees. Insectes Soc. 65, 419-429, (2018).

$111885 \quad$ Nayfach, S., Rodriguez-Mueller, B., Garud, N. \& Pollard, K. S. An integrated metagenomics pipeline for strain profiling reveals novel patterns of bacterial transmission and biogeography. Genome Res. 26, 1612-1625, (2016).

$112186 \quad$ Kim, D., Paggi, J. M., Park, C., Bennett, C. \& Salzberg, S. L. Graph-based genome alignment and genotyping with HISAT2 and HISAT-genotype. Nat. Biotechnol. 37, 907-915,

$112487 \quad$ Anders, S., Pyl, P. T. \& Huber, W. HTSeq--a Python framework to work with high1125 throughput sequencing data. Bioinformatics 31, 166-169, (2015).

$112688 \quad$ Love, M. I., Huber, W. \& Anders, S. Moderated estimation of fold change and 1127 dispersion for RNA-seq data with DESeq2. Genome Biol. 15, 550, (2014).

$1128 \quad 89 \quad$ Benjamini, Y. \& Yekutieti, D. The control of the false discovery rate in multiple 1129 testing under dependency. Ann. Statist. 29, 1165-1188, (2001).

$113090 \quad$ Huerta-Cepas, J. et al. Fast genome-wide functional annotation through orthology 1131 assignment by eggNOG-mapper. Mol. Biol. Evol. 34, 2115-2122, (2017). 
113291 Yu, G., Wang, L. G., Han, Y. \& He, Q. Y. clusterProfiler: an R package for comparing biological themes among gene clusters. OMICS 16, 284-287, (2012).

113492 Shen, S. et al. rMATS: robust and flexible detection of differential alternative splicing 1135 from replicate RNA-Seq data. Proc. Natl. Acad. Sci. U S A 111, E5593-E5601, (2014).

$113693 \quad$ Altschul, S. F., Gish, W., Miller, W., Myers, E. W. \& Lipman, D. J. Basic local 1137 alignment search tool. J. Mol. Biol. 215, 403-410, (1990).

113894 Meng, L. et al. Proteomics reveals the molecular underpinnings of stronger learning 1139 and memory in eastern compared to western bees. Mol. Cell Proteomics 17, 255-269, (2018).

114095 Wong, J. M. et al. Benzoyl chloride derivatization with liquid chromatography-mass 1141 spectrometry for targeted metabolomics of neurochemicals in biological samples. $J$.

1142 Chromatogr. A. 1446, 78-90, (2016).

114396 MacLean, B. et al. Skyline: an open source document editor for creating and analyzing 1144 targeted proteomics experiments. Bioinformatics 26, 966-968, (2010).

114597 Chong, J., Wishart, D. S. \& Xia, J. Using MetaboAnalyst 4.0 for comprehensive and 1146 integrative metabolomics data analysis. Curr. Protoc. Bioinformatics 68, e86, (2019).

114798 Langfelder, P. \& Horvath, S. WGCNA: an R package for weighted correlation 1148 network analysis. BMC Bioinformatics 9, 559, (2008).

114999 Shannon, P. et al. Cytoscape: a software environment for integrated models of 1150 biomolecular interaction networks. Genome Res. 13, 2498-2504, (2003).

\section{Acknowledgments}

This work was founded by National Key R\&D Program of China, (Grant No. 2019YFA0906500), National Natural Science Foundation of China Project 31870472.

\section{Author Contributions}

H.Z. supervised the study; H.Z. and Z.Z. designed the study; Z.Z., Q.C. and Y.S. 1157 collected samples and performed the behavioral experiments; Z.Z. generated data and 1158 performed the data analyses with contributions from X.M. and X.H.; H.Z., Z.Z., X.M., and 1159 X.H. prepared the manuscript.

\section{Competing Interests}

1161 The authors declare no competing interests. 